\title{
Live Exploration of Dynamic Rings
}

\author{
G. Di Luna* S. Dobrev $\stackrel{\dagger}{\dagger}$ P. Flocchini $\stackrel{\ddagger}{\ddagger}$ N. Santoro ${ }^{\S}$
}

October 12, 2018

\begin{abstract}
In the graph exploration problem, a team of mobile computational entities, called agents, arbitrarily positioned at some nodes of a graph, must cooperate so that each node is eventually visited by at least one agent. In the literature, the main focus has been on graphs that are static; that is, the topology is either invariant in time or subject to localized changes.

The few studies on exploration of dynamic graphs have been almost all limited to the centralized case (i.e., assuming complete a priori knowledge of the changes and the times of their occurrence).

We investigate the decentralized exploration of dynamic graphs (i.e., when the agents are unaware of the location and timing of the changes) focusing, in this paper, on dynamic systems whose underlying graph is a ring.

We first consider the fully-synchronous systems traditionally assumed in the literature; i.e., all agents are active at each time step. We then introduce the notion of semi-synchronous systems, where only a subset of agents might be active at each time step (the choice of the subset is made by an adversary); this model is common in the context of mobile agents in continuous spaces but has never been studied before for agents moving in graphs. Our main focus is on the impact that the level of synchrony as well as other factors such as anonymity, knowledge of the size of the ring, and chirality (i.e., common orientation) have on the solvability of the problem, focusing on the minimum number of agents necessary.

We draw an extensive map of feasibility, and of complexity in terms of minimum number of agent movements. All our sufficiency proofs are constructive, and almost all our solution protocols are asymptotically optimal.
\end{abstract}

\section{Introduction}

\section{$1.1 \quad$ Framework}

\subsubsection{Graph Exploration}

The problem of graph exploration requires a team of mobile computational entities, usually called agents or robots, located at the nodes of a graph and capable of moving from node to neighbouring node, to explore the graph, with the requirement that each node is eventually visited by at least one agent.

This classical problem has been extensively investigated, starting from the pioneering work of Shannon [44. In the vast literature on the subject (e.g., see [1, 17, $18,23,28,32,43]$ ), a wide spectrum of different assumptions have been made and examined e.g. with regard to: the computational power of the agent(s); the structure of the graph and its properties; the level of topological knowledge available to the agents; whether or not the network is anonymous (i.e., the nodes lack distinct identifiers); whether the nodes can be marked (e.g., by leaving a pebble); the level of synchronization. In case of multiple agents, different types of means of communication have been considered, including: face-to-face, where agents communicate when they are on

\footnotetext{
*University of Ottawa, gdiluna@uottowa.ca, g.a.diluna@gmail.com

†Slovak Academy of Sciences, stefan.dobrev@savba.sk

$\ddagger$ University of Ottawa, flocchin@site.uottawa.ca

$\S$ Carleton University, santoro@scs.carleton.ca
} 
the same node; wireless, where the agents are able to communicate even when they are on different nodes; with whiteboard, where the agents communicate by writing on and reading from whiteboards present in each node.

Regardless of their differences, all these investigations share the common assumption that the graph is static: its topological structure does not change during the exploration. This is true also for those investigations that consider faulty nodes and/or faulty links (e.g., see [6, 16, 24,25]).

\subsubsection{Dynamic Graphs}

In distributed computing, researchers recently started to investigate highly dynamic graphs, that is graphs where the topological changes are not localized and sporadic; on the contrary, the topology changes continuously and at unpredictable locations. These investigations have been motivated by the development of highly dynamic networks, where changes are not anomalies (e.g., faults) but rather integral part of the nature of the system. These highly dynamic networks are modelled in a natural way in terms of time-varying graphs, a model formally defined in [13, where main classes of systems studied in the literature and their computational relationship were identified. If time is discrete (e.g., changes occur in rounds), the evolution of these systems can be equivalently described as a sequence of static graphs, called evolving graph, a model suggested in 34 and formalized in 27].

The study of distributed computations in highly dynamic graphs has focused mainly on problems of information diffusion and reachability, such as broadcast, routing, etc. (e.g., see [5,7, 9 12, 15, 33]), and on

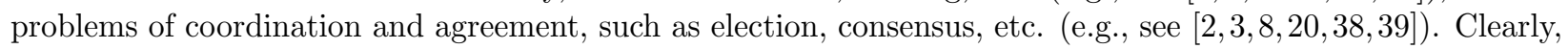
all these studies make strong assumptions in order to restrict the universe of the possible topological changes and their temporal occurrence. One such restriction is that topological changes are periodic (Class 8 of [13]), such as in carrier graphs (e.g., see $9,29,30,36,40]$ ). A popular restriction is by assuming that the network is always connected (Class 9 of [13]): at each time instant, there is a connected spanning subgraph; further assuming that such connected spanning subgraph persists for $T \geq 1$ time units defines the extensively studied sub-class of T-interval-connected systems (e.g., see $19,38,39,42$ ).

\subsubsection{Exploration of Dynamic Graphs}

Returning to the exploration problem, very little is known in the case of dynamic graphs. On the probabilistic side, there is an early seminal work on random walks [4. On the deterministic side there are: the study of the complexity of computing a foremost exploration schedule under the 1-interval-connectivity assumption [41, generalized and extended in 26]; the computation of an exploration schedule for rings under the T-intervalconnectivity assumption 37; and the computation of an exploration schedule for cactuses under the 1-intervalconnected assumption 35]. All these studies are however mainly centralized (or off-line, post-mortem); that is, they assume that the exploring agents have complete a priori knowledge of the topological changes and the times of their occurrence.

Very little is known on the distributed (or on-line, live) case, i.e. when the location and timing of the changes are unknown to the agents. Exploration of carrier networks, a periodic class of time varying graphs, by a synchronous agent, has been studied in 30 , where the feasibility of the problem is investigated depending on the knowledge available to the agent (size of the network or upper bound, length of the period) and where optimal solutions are proposed. Under a slightly different model, similar results can be found also in [36]. Exploration has also been examined assuming that the dynamic graph is $\delta$-recurrent (i.e., each edge appears at least once every $\delta$ rounds) 37]. Apart from these results, to the best of our knowledge, nothing is known.

\subsection{Contributions}

In this paper, we investigate the distributed exploration of dynamic rings under the 1-interval connectivity assumption by mobile agents without explicit means of communication.

We consider three different termination requirements once the ring has been explored: Explicit Termination, where, within finite time, all agents must explicitly terminate and stop moving; Explicit Partial Termination, 


\begin{tabular}{|c|l|l|l|}
\hline N. Agents & Assumptions & Even if & Result \\
\hline \hline 2 & No knolwedge on $n$, No Landmark & Non-anonymous agents, Chirality & $\begin{array}{l}\text { Partial Termination Impossible } \\
\text { (Th. 1 } 1 \text { I }\end{array}$ \\
\hline Any & $\begin{array}{l}\text { No knolwedge on } n, \text { No Landmark, } \\
\text { Anonymous agents }\end{array}$ & Chirality & $\begin{array}{l}\text { Partial Termination Impossible } \\
(\text { Th. 2p }\end{array}$ \\
\hline
\end{tabular}

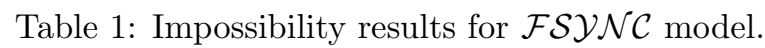

\begin{tabular}{|c|l|l|}
\hline N. Agents & Assumptions & Exploration with Termination \\
\hline \hline 2 & Known bound $N$ & Explicit Termination in time 3N-6 (Th. 3) 3$)$ \\
\hline \hline 2 & Chirality, Landmark & Explicit Termination in time $O(n)($ Th. 6 \\
\hline 2 & Landmark & Explicit Termination in time $O(n \log (n))($ Th. 8$)$ \\
\hline
\end{tabular}

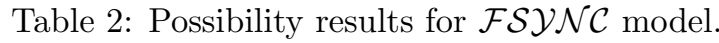

where, within finite time, at least one agent explicitly terminates and stops moving; Unconscious Exploration, where the agents are not required to stop.

We first consider the fully-synchronous systems (Section 3) traditionally assumed in the literature; i.e., all agents are active at every time step. We then introduce the notion of semi-synchronous systems (Section 4), where at each time step only a subset of the agents might be active (the choice of the subset is made by an adversary). The semi-synchronous model is common in the context of mobile agents in continuous spaces (e.g., [31]) but has never been studied before for agents moving in graphs. Our main focus is on the impact that the level of synchrony as well as other factors such as knowledge of the size of the ring, chirality (i.e., common sense of orientation), and anonymity, have on the solvability of the problem.

We start by examining the exploration problem in fully synchronous systems $(\mathcal{F} \mathcal{S Y N C})$, with two agents, after showing that it is unsolvable with only one.

For anonymous rings, we first show that, without any additional knowledge on the ring size (e.g., an upperbound), two non-anonymous agents cannot explore and partially terminate. If the agents are anonymous, the same impossibility holds for any number of agents. On the other hand, unconscious exploration is possible using two anonymous agents and no additional knowledge.

If there is knowledge of an upper bound $N$ on the ring size, we show that two anonymous agents can explore and they can both terminate in $3 N-6$ rounds. This can be done even if agents do not have a common chirality.

For non-anonymous rings, we show that the presence of a single observably different node (landmark) allows two anonymous agents to solve the exploration problem with explicit termination. This can be done without the need of any additional information. We provide an algorithm that terminates in $O(n)$ rounds when there is chirality, and an $O(n \log (n))$ algorithm for the case without chirality. For the case of no chirality we do not know if $O(n \log (n))$ rounds are necessay.

A summary of these results is shown in Tables 1 and 2, All the sufficiency proofs of $\mathcal{F S Y \mathcal { N C }}$ are constructive and, apart for the algorithm for the case of non-anonymous ring and no chirality, all the proposed algorithms are asymptotically optimal.

We then examine the problem in semi-synchronous systems $(\mathcal{S} \mathcal{S Y N C})$. In these systems it is possible that an agent $a$ waiting to traverse a missing link $e$ is inactive in the round the edge reappears. Depending on what happens to that agent, we consider and analyze three different transportation models (described in details later in the paper) and establish feasibility and complexity results:

- No Simultaneity (NS) model : $a$ is not allowed to move while inactive. This is the weakest of the models that we consider. In this case, exploration is impossible with any number of agents, even with exact knowledge of the ring size, nodes with distinct IDs, and common chirality.

- Passive Transport model (PT): $a$ is transported on $e$. In this case, we show that, without chirality, two anonymous robots are not sufficient to explore the ring; the result holds even if there is a distinguished landmark node and the exact network size is known. On the other hand, with chirality, two agents can 


\begin{tabular}{|c|c|l|l|l|}
\hline Model & N. Agents & Assumptions & Even if & Result \\
\hline \hline (NS) & Any & None & $\begin{array}{l}\text { Chirality, Known } n \text {, Landmark, Non- } \\
\text { anonymous Agents }\end{array}$ & Exploration impossible (Th. 9) \\
\hline \hline \multirow{2}{*}{ (PT) } & 2 & $\begin{array}{l}\text { No Chirality, } \\
\text { Anonymous } \\
\text { Agents }\end{array}$ & Known $n$, Landmark & Exploration impossible (Th. 10 \\
\cline { 2 - 5 } & 2 & None & Chirality, Known $n$, Landmark & $\begin{array}{l}\text { Explicit Termination impossible (Th. } \\
\text { 11) }\end{array}$ \\
\hline \hline ( ET) & Any & Unknown $n$ & $\begin{array}{l}\text { Known bound } N, \text { Chirality, Landmark, } \\
\text { Non-anonymous Agents }\end{array}$ & $\begin{array}{l}\text { Partial Termination impossible (Th. } \\
19)\end{array}$ \\
\hline
\end{tabular}

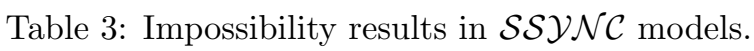

\begin{tabular}{|c|c|c|c|}
\hline Model & N. Agents & Assumptions & Exploration and Termination \\
\hline \multirow{4}{*}{$(\mathrm{PT})$} & \multirow{2}{*}{2} & Chirality, Known bound $N$ & Partial Termination in $O\left(N^{2}\right)$ moves (Th. 12 \\
\hline & & Chirality, Landmark & Partial Termination in $O\left(n^{2}\right)$ moves (Th. 14$)$ \\
\hline & \multirow{2}{*}{3} & Known bound $N$ & Partial Termination in $O\left(N^{2}\right)$ moves (Th. \\
\hline & & Landmark & Partial Termination in $O\left(n^{2}\right)$ moves (Th. 17 ) \\
\hline \multirow{2}{*}{$(\mathrm{ET})$} & 2 & Chirality & Unconscious Exploration possible (Th. 18] \\
\hline & 3 & Known $n$ & Partial Termination possible (Th. 20 ) \\
\hline
\end{tabular}

Table 4: Possibility results for $\mathcal{S} \mathcal{S Y N C}$ models.

perform the exploration if there is a known upper bound on the ring size or there is a landmark node. As for termination, we show that it is impossible to guarantee explicit termination of both agents (even if exact knowledge of the size, chirality and landmark are available). On the other hand, we prove that it is always possible for at least one of the agents to terminate. Interestingly, presence of chirality allows to solve the problem with only 2 agents; without chirality 3 agents are necessary. The PT model is the strongest of the $\mathcal{S} \mathcal{S} \mathcal{N} \mathcal{N}$ models.

- Eventual Transport (ET) model: $a$ is not allowed to move while inactive but, should the edge be present for an infinite number of rounds, $a$ is guaranteed to be eventually active at a round when the edge is present. In this case, we show that exploration with partial termination of all agents is impossible, regardless of the number of agents, even if an upper bound on the ring size is known, nodes have distinct IDs, and agree on chirality. On the other hand, with exact knowledge of the ring size, we prove that exploration is possible with three anonymous agents even without chirality, and at least one agent explicitly terminates.

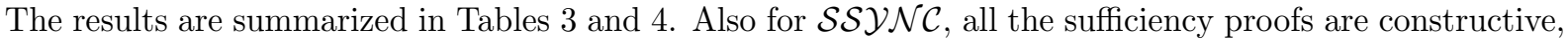
and almost all the proposed algorithms are asymptotically optimal.

\section{Model and Basic Limitations}

\subsection{Model and Terminology}

Let $\mathcal{R}=\left(v_{0}, \ldots v_{n-1}\right)$ be a synchronous ring where, at any time step $t \in N$, one of its edges might not be present; the choice on which link is missing (if any) is made by an adversary. Such a dynamic network is known in the literature as a 1-interval connected ring.

Each node $v_{i}$ is connected to its two neighbours $v_{i-1}$ and $v_{i+1}$ via distinctly labeled ports $q_{i-}$ and $q_{i+}$, respectively (all operations on the indices are modulo $n$ ); the labeling of the ports may not be globally consistent, thus might not provide an orientation, and the label may not be comparable 14. The ring is said to be anonymous if the nodes have no distinguishable identifiers, and with landmark if there is a node (the landmark) which is different from all others.

Operating in $\mathcal{R}$ is a set $A=\left\{a_{0}, \ldots, a_{m-1}\right\}$ of agents, each provided with memory and computational capabilities. The agents are anonymous and all execute the same protocol. Any number of agents can reside 
at a node at the same time. Initially located at arbitrary nodes, not necessarily distinct, they do not have any explicit communication mechanism, nor can leave marks on the nodes. The agents are mobile, that is they can move from node to neighboring node. To move, an agent has to position itself on the port from which it wants to leave. Access to ports is done in mutual exclusion: an agent will not succeed to gain an already occupied port; when several agents try to position themselves on the same port, only one of them succeeds. Two agents moving in opposite directions on the same edge in the same round might not be able to detect each other.

Each agent $a_{j}$ has a consistent private orientation of the ring; that is, it has a function $\lambda_{j}$ which designates each port either left or right and $\lambda_{j}\left(q_{i-}\right)=\lambda_{j}\left(q_{k-}\right)$, for all $0 \leq i, k<n$. The orientations of the agents might not be the same. If all agents agree on the same orientation and are aware of it, we say that there is chirality.

The system operates in synchronous time steps, called rounds. Initially, all agents are inactive. Each time step $t \in N$ starts with a non-empty subset $A(t) \subseteq A$ of the agents becoming active. Upon activation, agent $a_{j} \in A(t)$ at node $v_{i}$ performs a sequence of operations: Look, Compute, and (possibly) Move.

1. Look: The agent determines its own position within the node (i.e., whether or not it is on a port, and if so on which one), and the position of the other agents (if any) at that node. We call this information a snapshot.

2. Compute: Based on the snapshot and the content of its local memory, the agent executes its protocol (the same for all agents) to determine whether or not to move and, if so, in what direction; the result will be direction $\in\{$ left, right, nil $\}$, where left and right are with respect to its own local orientation. If direction $=n i l$, the agent becomes inactive. If direction $\neq n i l, a_{j}$ attempts to access the appropriate port (if not already there); if it gains access, it positions itself on the port, otherwise it sets private variable moved $=$ false and becomes inactive.

3. Move: Let the agent be positioned on port $q_{i-}$ (resp., $q_{i+}$ ) after computing. If the link between $v_{i}$ and $v_{i-1}$ (resp., $v_{i+1}$ ) is present in this round, then agent $a_{j}$ will move to $v_{i-1}$ (resp., $v_{i+1}$ ), reach it, set private variable moved $=$ true, and become inactive. If the link between $v_{i}$ and $v_{i-1}$ (resp., $v_{i+1}$ ) is not present, then agent $a_{j}$ will remain in the port, set moved $=$ false, and become inactive. In either case, access to port $q_{i-}$ (resp., $q_{i+}$ ) continues to be denied to any other requesting agent during this round.

By definition, the delays are such that all active agents have become inactive by the end of round $t$; the system then starts the new round $t+1$.

Notice that, since access to a port is in mutual exclusion, in the same round at most one agent will move in each direction on the same edge. Recall that two agents moving in opposite directions on the same edge in the same round might not be able to detect each other.

A major computational factor is the nature of the activation schedule of the agents. If $A(t)=A$ for all $t \in N$, that is all agents are activated at every time step, the system is said to be fully synchronous $(\mathcal{F S Y N C})$. Otherwise the system is said to be semi-synchronous $(\mathcal{S S Y N \mathcal { C }})$; the agents that are not activated in a round are said to be sleeping or passive in that round; the choice of which agent is active in a round is made under an adversarial scheduler, where every agent is activated infinitely often. When an agent is activated, it does not know whether or not it was active in the previous round. Observe that in $\mathcal{S S Y \mathcal { N C }}$ it is possible for an agent to be sleeping on a port. This is indeed the case when an agent $a$ gains access to a port $q$ when the link is not there (thus, it remains on $q$ ), and $a$ is not activated in the next round. What may happen to an agent sleeping on a port gives raise to different models, described in the following:

- No Simultaneity (NS): A sleeping agent cannot move. There is no guarantee of simultaneity for an agent sleeping on a port.

- Passive Transport (PT): If an agent is sleeping on a port at round $t$ and the corresponding edge is present in that round, the agent is moved to the other endpoint of the edge in round $t$.

- Eventual Transport (ET): A sleeping agent cannot move. If an agent is sleeping on a port at round $t$ and the corresponding edge is present infinitely many times, then the agent will eventually become active at a round $t^{\prime}>t$ when the corresponding edge is present (simultaneity condition). 
The algorithm executed by the agents solves the exploration problem if, within finite time, every node of the ring is visited by at least one agent. The exploration is said to be with explicit termination if every agent executing the algorithm within finite time enters a terminal state and no longer moves. The terminal state has to be entered only after the exploration of the ring. The exploration is said to be with explicit partial termination (or just partial termination) if at least one agent executing the algorithm enters a terminal state within finite time and no longer moves. Finally, it is said to be unconscious if the agents are not required to stop nor to be aware that the ring has been visited.

\subsection{Basic Limitations}

We begin our study by showing simple impossibility results.

Observation 1. The adversary can prevent an agent from leaving the initial node $v_{0}$, by always removing the edge over which the agent wants to leave $v_{0}$.

From this Observation, we immediately get:

Corollary 1. A single agent is not able to explore the ring.

Hence, at least two agents are needed. However, the adversary can prevent their cooperation:

Observation 2. The adversary can prevent two agents starting at different locations from meeting each other even if they have unlimited memory, common chirality and distinct known IDs. This result holds even

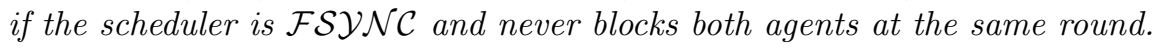

Proof. The adversary will never remove an edge, except in the case when that would lead to agents meeting in the next step. There are two possible cases how the agents can meet in the next step.

Case 1: One agent is waiting at a node and the other agent, at a neighbouring node, decides to traverse the edge $e$ connecting the two nodes. In this case, the adversary removes edge $e$.

Case 2:Both agents decide to traverse different edges $e$ and $e^{\prime}$ leading to the same vertex. Again, it is sufficient for the adversary to remove one of the two edges to prevent rendezvous.

Theorem 1. There does not exist any partially terminating deterministic exploration algorithm of anonymous rings of unknown size by two agents, even with distinct IDs, common chirality, and when the scheduler is FSYNC.

Proof. By contradiction, assume that there exists a terminating exploration algorithm $\mathcal{A}$. Let us consider an execution $E$ of $\mathcal{A}$ on a dynamic ring of size $n$, where agents $a$ and $b$ start in two distinct locations and where the adversary always prevents the meeting of the agents, never blocking the agents at the same round. By Observation 2 this run exists.

Let us assume, without loss of generality, that in such an execution agent $a$ is the first one terminating at round $r(E)$. Let us now consider an execution $E^{\prime}$ of $\mathcal{A}$ on a dynamic ring of size $n^{\prime}=8 r(E)$, where the agents start at two distinct locations at distance $4 r(E)$. The execution $E^{\prime}$ is constructed in such a way that, until round $r(E)$ neither agent can distinguish this execution from $E$. This is possible since in $E$ the adversary never blocks the two agents at the same time and they do not meet.

Since the size of the ring is $8 r(E)$ and the agents started $4 r(E)$ apart, at round $r(E)$ (when agent $a$ terminates) the distance between the two agents is at least $2 r(E)$ and there are at least $6 r(E)$ unexplored nodes. The execution $E^{\prime}$ is completed by the adversary blocking agent $b$ at round $r(E)$ and afterwards, preventing it from exploring the unexplored nodes. Therefore, in this execution, the partial termination of algorithm $\mathcal{A}$ is incorrect.

Observe now that for anonymous agents the impossibility of explicit termination holds regardless of their number. This is because, in the setting when there is orientation and no edge is removed, all the agents will act in the same way at each time step. If one were to decide to terminate after $t$ time steps, they all would do so at the same time; however, since they do not know the ring size, they would do the same also in a ring of size $n$ multiple of $t$, terminating without having completed the exploration. Indeed, with the same reasoning, it is immediate that even the weaker partial termination is impossible. 
Theorem 2. There does not exist any partially terminating deterministic exploration algorithm of anonymous rings of unknown size by anonymous agents, regardless of their number. The result holds even if agents have

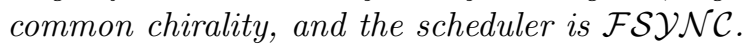

Summarizing, without some knowledge of the size of the ring or without the asymmetry introduced by a landmark node, exploration with partial termination is impossible even in the fully synchronous model by two agents with IDs, or by any number of anonymous agents. Notice that Theorems 1 and 2 would hold even if the agents were equipped with wireless communication.

As for the amount of time required for exploration, there exists the following lower bound due to [26]:

Observation 3. [26] Exploration of an anonymous ring by two anonymous agents requires at least $2 n-3$ time in the worst case, even if there is chirality and the scheduler is $\mathcal{F} \mathcal{S Y N C}$.

\section{$3 \quad$ Ring Exploration in $\mathcal{F S Y \mathcal { N C }}$}

In this section, we consider exploration when the system is fully synchronous, presenting and analyzing protocols that solve the problem under different assumptions on knowledge of the ring size, anonymity of the nodes, and presence of chirality. All these solutions do not require the agents to be able to communicate explicitly.

Our algorithms use as a building block procedure Explore $\left(\operatorname{dir} \mid p_{1}: s_{1} ; p_{2}: s_{2} ; \ldots ; p_{k}: s_{k}\right)$, where dir is either left or right, $p_{i}$ is a predicate, and $s_{i}$ is a state. In Procedure EXPLORE, the agent performs Look, then evaluates the predicates $p_{1}, \ldots, p_{k}$ in order; as soon as a predicate is satisfied, say $p_{i}$, the procedure exits and the agent does a transition to the specified state, say $s_{i}$. If no predicate is satisfied, the agent tries to Move in the specified direction dir and the procedure is executed again in the next round.

Furthermore, the following variables are maintained by the algorithms:

- Ttime, Tsteps: the total number of rounds and edge traversals, respectively, since the beginning of the execution of the algorithm.

- Etime, Esteps: the total number of rounds and edge traversals, respectively, since the last call of procedure EXPLORE.

- Btime: the number of consecutive rounds the agent has been currently waiting in a port.

In particular, the following predicates are used:

- meeting: both agents are in the node.

- catches: the agent observes the other agent on the port corresponding to its moving direction.

- caught: the agent is on the port after a failed move, the other agent is observed in the node.

Observe that, in a fully synchronous system, when predicate catches holds for an agent, then caught holds for the other agent. In the following, we say that the agents catch each other if both predicates hold.

\subsection{Known Upper Bound on Ring Size}

In this section we study the simple case of exploring the ring when the agents know an upper-bound $N \geq n$ on the ring size, and we show how to solve the problem in asymptotically optimal time, even without chirality.

The directions left and right now refer to the local orientation of the individual agent. The algorithm is shown in Figure 1. We use the predicate failed, that is verified when an agent tries to enter a port and it fails to do so.

The algorithm works as follows. At the beginning, each agent goes left; recall that the left direction could be different for the two agents. An agent keeps going left unless: $i$ ) it catches the other agent in the first $2 N-4$ rounds; or ii) $2 N-4$ rounds have passed and the agent has been blocked for $N-1$ rounds; or iii) it 
States: \{Init, Bounce, Forward, Terminate\}.

In state Init:

ExplORE (left $\mid$ (Ttime $\geq 2 N-4 \wedge$ Btime $=N-1) \vee$ failed: Bounce; catches: Bounce; caught:

Forward; Ttime $\geq 2 N-4$ : Forward)

In state Bounce:

EXPLORE(right $\mid$ Ttime $\geq 3 N-6$ : Terminate)

In state Forward:

EXPLORE(left $\mid$ Ttime $\geq 3 N-6$ : Terminate)

Figure 1: Algorithm KnownNNoChiRALITY

is in a node and it fails to enter a port. In all these cases, the agent changes state to Bounce and it goes right until termination, at round $3 N-6$.

If instead an agent is caught in the first $2 N-4$ rounds, then it enters in state Forward and it keeps the left direction until termination, at round $3 N-6$.

Theorem 3. Algorithm KNOWNNNoCHIRALITY allows two anonymous agents without chirality to explore a 1-interval connected ring and to explicitly terminate in time $3 N-6$, where $N$ is a known upper-bound on the ring size.

Proof. The termination by round $3 N-6$ is trivial for the condition Ttime $\geq 3 N-6$. It is sufficient to show that the ring has been explored when Ttime $=3 N-6$. Let $a, b$ be the two agents.

- We first examine the case where $a, b$ start on the same node $v$.

If they have different agreements on the left direction then no agent can change direction in the first $2 N-4$ rounds. It is trivial to see that after at most $N-1$ rounds the agents have explored the ring, and hence the Theorem holds.

Let us consider then the case in which the agents agree on the same left direction, and hence try to traverse the same edge $e$. Since the access to a port is done in mutual exclusion, only one of them will enter the port, while the other will fail. If $e$ is not missing at round 1, then at the beginning of round 2 the two agents will be in different nodes; otherwise they will be on the same node. In either case, for one of the two agents the predicate failed is true; notice that the failed predicate will not be verified in any other case. This implies that the two agents will have different directions, and they will not change such directions in the first $2 N-4$ rounds; thus in the next $N-1$ rounds the ring will be explored. Hence the Theorem holds.

- We now consider when $a$ and $b$ start on different nodes.

Let us examine the case when they disagree on the left direction. After $N-3$ rounds, they either (i) are at distance 2 , or (ii) are at distance 1, or (iii) crossed each other, where the distance is the number of edges in the portion of the ring pointed by the left directions of the agents. (i) If the distance between the agents is 2 , then they were initially on two neighbouring nodes; therefore, in the next round, at time $N-2$, the ring will be explored. (ii) If they are at distance 1 and the edge between them is not missing for the successive $N-1$ rounds, then they will cross each other and the ring will be explored by at most round $3 N-6$. If the edge between them is missing for the successive $N-1$ rounds, then at round $2 N-4$ they will switch direction and the ring will be explored in the next $N-2$ rounds. (iii) If they cross each other on edge $e$, they will not change direction for the successive $N-1$ rounds, thus they will explore the ring. That is, in all three cases the Theorem holds.

Finally, let us consider the case when they agree on the left direction. 


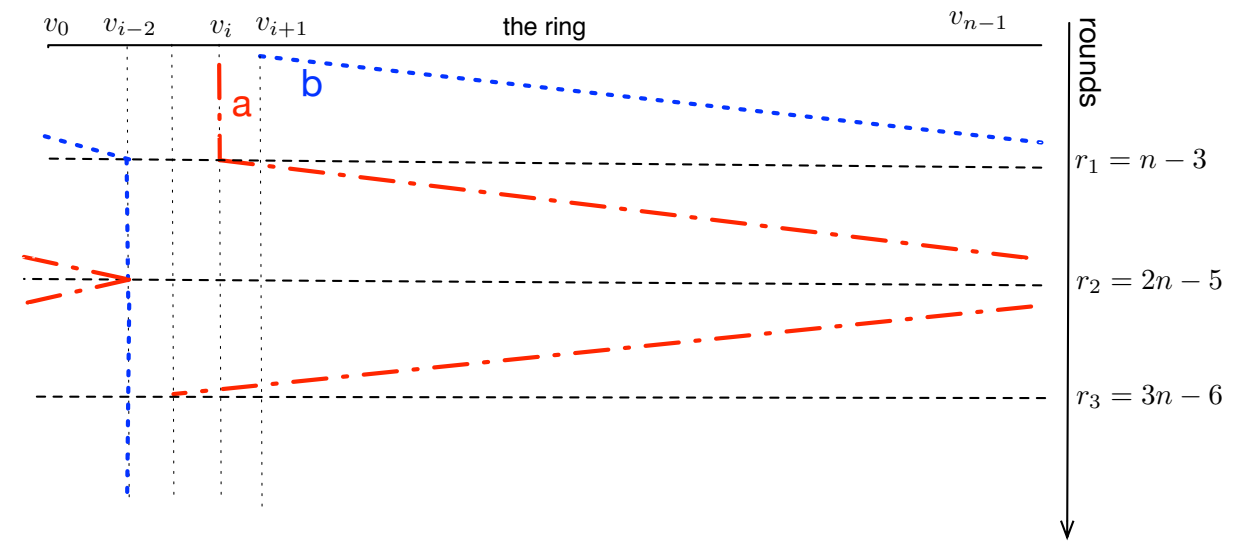

Figure 2: Schedule where Algorithm KnownNNoChiraliTy takes $3 n-6$ rounds to explore.

If they catch each other before round $2 N-4$, then the ring will be explored in the next $N-1$ rounds terminating the exploration by round $3 N-6$.

If they do not catch each other, then at each round at least one of them will traverse an edge, since they cannot be blocked at the same time. Let us suppose that $a$ traverses $N-k$ edges with $k<2$; then $b$ traversed at least $N-4+k \geq N-1$ edges, exploring the ring. So the last remaining case is when agent $a$ traverses exactly $N-2$ edges; but this implies that also $b$ has traversed at least $N-2$ edges and, since they start from different nodes, the ring has been explored also in this case.

Notice that there exists a schedule in which the exploration takes $3 n-6$ rounds, so the cost is tight for $N=n$. The schedule is reported in the Figure 2 the agents start on distinct nodes, $a$ on $v_{i}$ and $b$ on $v_{i+1}$, and there is chirality. Agent $a$ is blocked for the first $n-3$ rounds; in the meanwhile agent $b$ reaches node $v_{i-2}$. At this point, $b$ is blocked on node $v_{i-2}$ until round $r_{2}=2 n-5$; during this time $a$ moves until it catches $b$; this happens exactly at round $r_{2}$. In the next $n-1$ rounds, $b$ is still blocked while $a$ reaches node $v_{i-1}$, exploring the ring.

The algorithm is asymptotically optimal, as shown by the following theorem, even with the lesser requirement of partial termination.

Theorem 4. Exploration with partial termination of an anonymous ring by two anonymous agents with knowledge of an upper bound $N$ on the ring size requires at least $N-1$ time in the worst case, even with chirality.

Proof. By contradiction, let $\mathcal{A}$ be a correct exploration algorithm that allows partial termination with knowledge of an upper bound $N$ in at most $N-2$ time unit. Let $R_{i}$ be an anonymous ring of size $i$, and for $N>5$ let $R(N)=\left\{R_{i}: 3 \leq i \leq N\right\}$. Consider now the simultaneous execution of $\mathcal{A}$ in $R(N)$ started by initially placing in each $R \in R(N)$ two agents with chirality at two neighbouring nodes, and then making no edge ever disappear.

Clearly, at each time step, all the agents in all rings of $R(N)$ have the same view, perform exactly the same movement in the same direction; furthermore, in each ring they are unaware of each other, and keep their distance to 1 . By symmetry, if one of the agents terminates in a ring at time $t$, then they all do in all the rings at the same time. By assumption, the execution of $\mathcal{A}$ terminates at a time $t \leq N-2$; this means that in $R_{N}$, when both agents terminate, at least one node is still unexplored at that time, contradicting the correctness of $\mathcal{A}$. 
States: $\{$ Init, Bounce, Reverse, Forward, Keep $\}$.

In state Init:

$G \leftarrow 2$, dir $\leftarrow$ left

Explore(dir; Etime $\geq 2 G \wedge$ Btime $>G$ : Reverse, Etime $\geq 2 G$ : Keep, catches: Bounce, caught:Forward)

$\underline{\text { In state Reverse: }}$

$F \leftarrow 2 \cdot G$, dir $\leftarrow$ opposite $($ dir $)$

Explore(dir; Etime $\geq 2 G \wedge$ Btime $>G$ : Reverse, Etime $\geq 2 G$ : Keep, catches: Bounce, caught:Forward)

In state Keep:

$G \leftarrow 2 \cdot G$

ExPlore(dir; Etime $\geq 2 G \wedge$ Btime $>G$ : Reverse, Etime $\geq 2 G$ : Keep, catches: Bounce, caught:Forward)

In state Bounce:

EXPLORE(opposite(dir))

In state Forward:

$\operatorname{EXPLORE}(d i r)$

Figure 3: Algorithm UnCONSCIOUs Exploration

\subsection{No Known Bounds On Ring Size}

We now consider exploring the ring when no upper-bound on its size is available to the agents. Under this condition, by Theorem 1, it is impossible for two agents to explore an anonymous ring with termination, even if the agents have unique IDs. Hence, for exploration to occur, either termination must not be required or the ring must not be anonymous. In the following we consider precisely those two cases. We first show how unconscious exploration can be performed without any other condition even if the agents are anonymous. We then consider a ring in which there is a special node, called landmark, different from the others and visible to the agents; we prove that exploration can be performed with termination, even if the agents are anonymous, in time $O(n)$ if there is chirality, $O(n \log n)$ otherwise.

\subsubsection{Unconscious Exploration}

We present a protocol, UNCONSCIOUS EXPLORATION, that allows two anonymous agents to perform exploration without knowing any bound on the ring size. The basic idea of the algorithm is for each agent to guess the size of the ring with an initial estimate $G$ and move in one direction for a time equal to twice the estimate; the agent will then double the size estimate. It changes direction if it has been blocked for a time that is equal to the previous estimate and it will keep direction otherwise. This process is repeated with the new guess. The algorithm is shown in Figure 3.

Theorem 5. Algorithm UnCONSCIOUs EXPLORATION allows two anonymous agents without chirality to explore, without terminating, an 1-interval connected ring; the exploration is completed in $O(n)$ time.

Proof. If the agents catch each other, then they start moving in opposite directions and, in the subsequent $n-1$ moves (unknown to them), they will explore the whole ring, proving the Theorem.

Consider now the case when the agents never catch each other. Let a phase be the period of time when the guess remains the same. Since $G$ is always doubled after $2 G$ time steps, at time $t_{n} \leq 4 n, G \geq n$. Let $r$ be the first round of the phase $P$ in which $G \geq n$.

If, at $r$, the agents are moving in the same direction, since they do not catch each other and in each time step at least one of them makes progress, in the next $2 G$ time steps the ring will be explored. 
Consider now the case when agents are moving in opposite directions: If at time $r+2 G-1$ neither of them is blocked on an edge, then by the next phase $P+1$ starting at round $r+2 G$, they must have crossed each other and they keep different directions; thus, the ring will be explored by the end of phase $P+1$. Otherwise, at time $r+2 G-1$ at least one of them has to be blocked on an edge $e$. At the beginning of the next phase, round $r+2 G$, three cases are possible:

- Only one agent changes direction; in this case, they will have the same direction in phase $P+1$, thus exploring in $O(n)$ rounds.

- Both agents reverse direction; this happens only if both have been blocked for the last $G$ rounds of phase $P$, thus they will change directions from two different endpoints of the same edge $e$, and the ring is explored by time $r+2 G+4 G$.

- No one changes direction; this implies that by the next $n$ rounds, i.e. by round $r+2 G+2 G$, they either crossed each other, and thus they will explore the ring by the end of phase $P+1$, or they are blocked on the endpoints of the same edge $e^{\prime}$. In the latter case, if the edge $e^{\prime}$ is removed for the last $2 G$ rounds of phase $P+1$, then at round $r+2 G+4 G$ they both change directions moving from the two endpoints of $e^{\prime}$, and thus exploring the ring by the end of phase $P+2$. Otherwise, if the edge is present for one round in the the last $2 G$ rounds of phase $P+1$, then they will cross each other and continue in the same direction for the phase $P+2$, exploring the ring.

\subsubsection{Termination: Landmark and Chirality}

We now focus on solutions with termination. By Theorem 1, in absence of bounds on the ring size, the ring cannot be anonymous. Hence, we assume that there is a special node, called landmark, different from the others and visible to the visiting agents. in this subsection we consider chirality, but no other additional knowledge, and we show that two anonymous agents can explore the ring and terminate in optimal time $O(n)$.

Let $v^{*}$ be the landmark, identifiable by the agents: when performing a Look operation at some node $v$, a flag IsLandmark is set to true if and only if $v=v^{*}$. The basic idea is to explore the ring using the landmark to compute the size and allow termination. In order to coordinate termination, the agents implicitly "communicate" when they catch each other (by waiting at the node if not sure whether to terminate, and by leaving it if they already know that the ring is explored). When the agents catch each other for the first time, they break symmetry and assume different roles.

We assign to them logical names: $F$ for the agent being caught, and $B$ for the one that caught $F$. These names do not change afterwards, even though it is possible for $F$ to catch $B$ later on.

Procedure LEXPLORE is very similar to EXPLORE with the following additions:

- Each agent keeps track of whether it is crossing the landmark and in which direction; furthermore, it tracks its distance from the landmark (since encountering it for the first time). In this way, it can detect whether it made a full loop around the ring. When it does so for the first time, variable size is set to the ring size $n$ (size is initialized to infinity, all the tests using it while it has this initial value will fail).

- An additional variable Ntime is maintained, tracking the total number of rounds since the agent learned $n$.

The complete pseudocode is shown in Figure 4. Both agents start going left. If they never meet, they terminate (see Lemma 1). If they catch each other, the naming is done. After naming, agent $F$ keeps going left. Agent $B$ moves right until either it completes a loop of the ring or it is blocked for a number of rounds larger than the number of edges it has traversed so far, i.e. predicate "Etime $>2$ Esteps". When one of these conditions is satisfied, agent $B$ goes left, and it tries to catch up with $F$. 
States: $\{$ Init, Bounce, Return, Forward, Terminate, BComm, FComm\}.

In state Init:

LEXPLORE(left | Ntime $>2$ size: Terminate; catches: Bounce; caught: Forward)

In state Bounce:

LEXPLORE(right $\mid$ meeting: Terminate; Etime $>2$ Esteps $\vee$ Ntime $>0$ : Return, catches: BComm)

In state Return:

bounceSteps $\leftarrow$ Esteps

LEXPLORE(left | Ntime $>3$ size $\vee$ caught: Terminate; catches: BComm)

In state Forward:

LEXPLORE $($ left $\mid$ Ntime $\geq 7$ size $\vee$ meeting $\vee$ catches: Terminate; caught: FComm)

In state BComm:

returnSteps $\leftarrow$ Esteps

if returnSteps $\leq 2 \cdot$ bounceSteps then

Move (right)

Terminate in the next round

else if you know that the ring is explored ( $n$ is known) then

Move (right)

Terminate in the next round

else

Stay for one round in the node

if agent $\mathrm{F}$ is in the node then

change state to Bounce and process it (in the same round) else

Terminate

$\triangleright$ agent F left, or tried to leave and is on the port - signalling to terminate

In state FComm:

if you know that the ring is explored ( $n$ is known) then

Move (left)

$\triangleright$ both must have waited on the same edge $\triangleright$ signal the need to terminate

Terminate in the next round else

Move from the port to the node

if agent $B$ is in the node then

Change state to Forward and process it (in the same round) else

Terminate

$\triangleright$ signal to $\mathrm{B}$ that $\mathrm{F}$ knows $n$

$\triangleright$ i.e. staying at the same node $\triangleright$ this happens next round

$\triangleright \mathrm{B}$ has left or is on the port

Figure 4: Algorithm LandmarkWithChirality 
If they catch up and $F$ has done less than Esteps steps to the left from its old position, then $B$ and $F$ have waited on the same edge, and hence the ring has been explored; $B$ can detect this, and it "communicates" the end of exploration to $F$.

If they catch up but $F$ has done at least Esteps steps to the left from its old position ( $B$ can detect this), they both keep executing the algorithm. Note that $F$ has made progress towards completing a ring loop, a condition that can be detected because of the landmark. Should such an event occur, then both $F$ and $B$ will eventually terminate.

If they do not catch up for a certain number of rounds, then they will both know that the ring is explored and they can terminate independently (see Lemma 2 ).

Lemma 1. In Algorithm LandmarkWithChirality, if the agents do not catch each other and stay in the Init state, then they will explore the ring and explicitly terminate by round $7 n-1$.

Proof. Let the agents do not catch each other and stay in the Init state.

We first show that two agents starting on different nodes will explore the ring and explicitly terminate by round $7 n-2$. Since the agents are moving in the same direction but they start from different nodes, in each round at least one of them makes progress. Since they do not catch each other, the difference between the number of successful moves by the agents is at most $n-1$. Therefore, if by round $5 n-2$ no agent has terminated, then both agents have crossed at least $2 n-1$ edges and hence they both know $n$. By construction, in additional $2 n$ steps, the agents will terminate. If an agent has terminated at round $r<5 n-2$, this means that at time $r-2 n$ this agent knew $n$, i.e. it has entered the landmark for the second time. As the agents did not catch each other, the other agent must have already entered the landmark. Since in the subsequent $2 n$ steps the agents do not catch each other and together made progress at least $2 n$ times, by round $r$ the other agent will enter the landmark for the second time, and by round $r+2 n$ it will terminate as well.

We examine now the case of the agents starting at the same node; since the agents are going in the same direction, at the first round they will both try to enter the same port. Since access to a port is granted in mutual exclusion, only one agent will succeed. If the edge $e$ corresponding to that port is missing then, at the beginning of next round, one agent sees the other inside the port after the failed move, and the agent in the port sees the other in the node. That is, the agents catch each other, contradicting the hypothesis.

Therefore, edge $e$ has to be present; this means that at the beginning of the next round the two agents will be in two different nodes.

But this is the same to consider as the agent are starting on a different nodes, hence we just have to add an additional round to the bound shown for the case of agents starting from distinct nodes.

Lemma 2. In Algorithm LANDMARKWithChirality, if an agent terminates, then the ring has been explored and the other agent will terminate as well.

Proof. We prove the lemma by case analysis on how the agents terminate. In the proof we assume that the left direction corresponds to a counter-clockwise direction. Consider first the case when the agents terminate at the same time. According to the algorithm, this happens only in the four cases considered below; in each case, we show that the ring has been explored:

1. Agent $F$, in state Forward, catches agent $B$ in state Return at node $w$ (see Figure 5): Consider the node $v$ where $B$ caught $F$ and changed state to Bounce the last time. The counter-clockwise segment from $v$ to $w$ has been explored by $F$, that never changed its direction. Consider now the node $z$ where $B$ changed state to Return the last time; it is not difficult to see that $z$ must be in the counter-clockwise segment from $v$ to $w$; this in turn implies that $B$ has explored the clockwise segment from $v$ to $w$. Thus the entire ring has been explored.

2. The agents, $F$ in state Forward and $B$ in state Bounce, moving in opposite direction meet at a node: The entire ring has clearly been explored.

3. Agent $F$, in state FComm, knows $n$ and signals $B$ to terminate: By construction. 


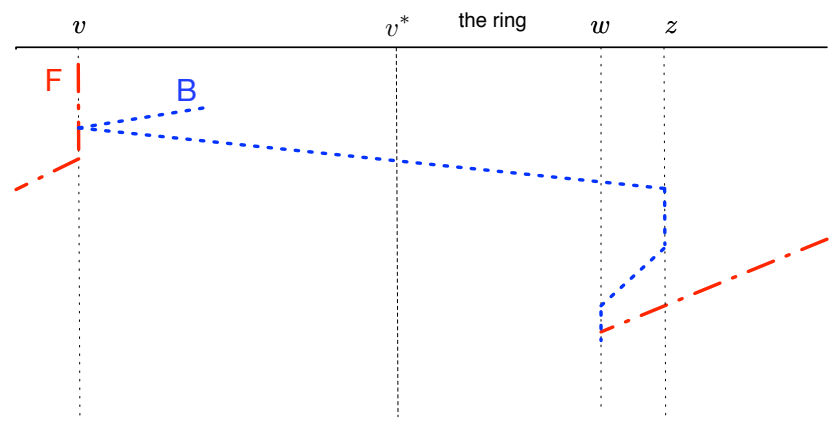

Figure 5: Agent $F$, in state Forward, catches agent $B$ in state Return at node $w$.

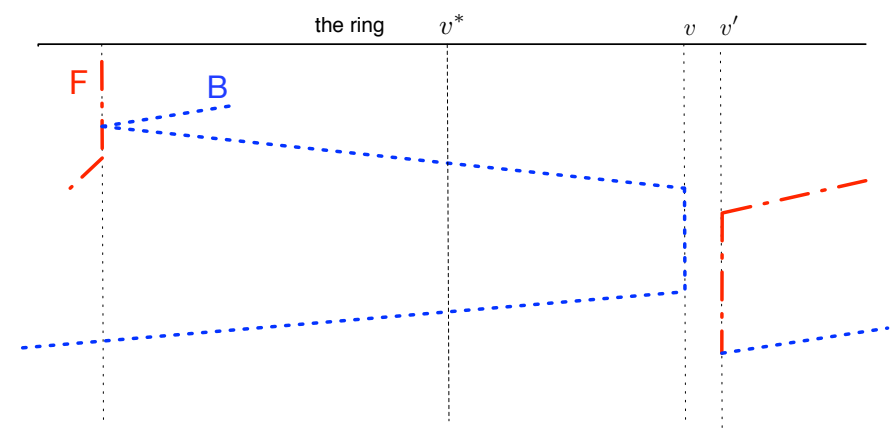

Figure 6: Agent $B$ signals $F$ to terminate, after checking that returnSteps $\leq 2 \cdot$ bounceSteps. This implies that both agents were waiting on two endpoints of the same edge, in this case edge $\left(v, v^{\prime}\right)$.

4. Agent $B$, in state $\mathrm{BComm}$, signals $F$ to terminate: According to the algorithm when this occurs either $B$ knows $n$, or returnSteps $\leq 2 \cdot$ bounceSteps. If $B$ knows $n$ the lemma trivially holds. Consider the second condition. When agent $B$ changes its direction, it has been blocked (not necessarily on the same edge) more than bounceSteps times. Satisfying the test returnSteps $\leq 2 \cdot$ bounceSteps means $F$ has either made progress of at most bounceSteps, or it has made one or more whole loops and then at most bounceSteps. In the first case $F$ has been blocked during one of the rounds when $B$ has been blocked; this can only happen if they had been blocked on the same edge, i.e. the ring has been explored (see Figure 6 for an example). In the latter case, the ring has obviously been explored.

Consider now the cases when one agent terminates first (the case of agents never meeting is handled by Lemma 11; we have two situations, in both we prove that the ring has been explored:

1. Agent $B$ terminates due to timeout Ntime $>3 n$. As the agents are moving in the same direction, the number of successful moves differs by at most $n-1$. Since in each time step at least one of them advances, in less than $3 n$ time steps from the moment when $B$ learned $n, F$ will also learn $n$ and eventually terminate.

2. Agent $F$ terminates due to timeout Ntime $\geq 7$.

Let $r$ be the round when $F$ learned $n$.

If $B$ entered state Bounce at round $r^{\prime} \leq r$, since the agents did not cross each other (satisfying the meeting predicate), agent $B$ switches to state Return at most $4 n-2$ rounds from round $r$, as we now show. In fact, at round $r+4 n-2$, we have Etime $\geq 4 n-2$. If Esteps $<2 n-1, B$ satisfies predicate 


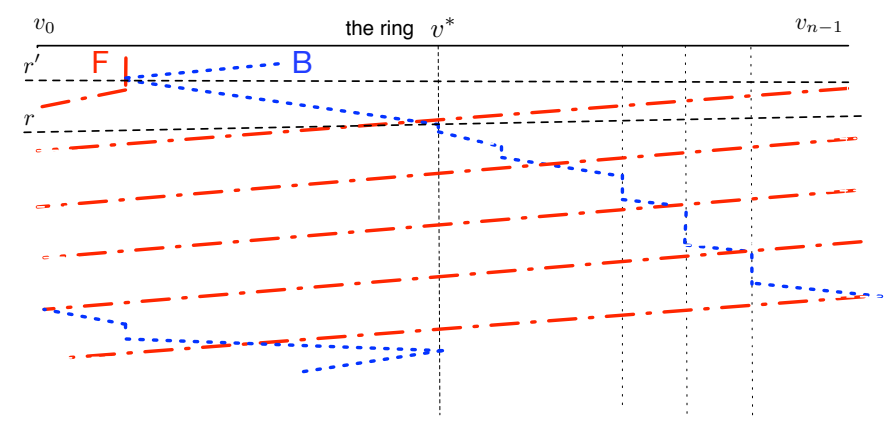

Figure 7: Agent $F$ terminates due to timeout Ntime $\geq 7 n$ : in this Figure we can see agent $F$ learning $n$ at round $r$, and agents $B$ switching state to Return after $4 n-2$ steps.

"Etime $>2$ Esteps" and thus enters state Return. On the other hand, if Esteps $\geq 2 n-1$, then $B$ has done at least a loop around the landmark $v^{*}$; therefore "Ntime $>0$ " and thus $B$ enters state Return, (see Figure 7 for an example). Now the analogous argument as for case 1 applies, both agents have the same direction and if $B$ catches $F$ it terminates. Therefore, if $B$ does not catch $F$, then it will learn $n$ with at most $3 n$ additional steps, and eventually terminate.

Notice that, if agent $B$ enters state Bounce after round $r, B$ will catch $F$ that would signal $B$ to terminate, proving the lemma. Otherwise, if $B$ does not catch $F$, that means that $B$ keeps staying in state Return after round $r$, then it will learn $n$ with at most $3 n$ additional steps, and eventually terminate.

Theorem 6. Algorithm LANDMARKWithCHIRALiTy allows two anonymous agents with chirality to explore a 1-interval connected ring with a landmark and to explicitly terminate in $O(n)$ time.

Proof. If the agents do not catch each other, the proof follows from Lemma 1. Consider now the case that the agents catch each other at least once. Also by Lemma 1, we know that the meeting will happen no later than in round $7 n-2$. The crucial observation is that, either the time between two consecutive meetings is linear in the progress made by agent $F$, or the agents terminate following the catch.

Let $p$ Time $_{i}$ denote the time between $i$-th and $i+1$-th catch and let forwdSteps $s_{i}$ be the progress made in that time by agent $F$. We have:

$$
\text { returnSteps }_{i}=\text { bounceSteps }_{i}+\text { forwdSteps }_{i}
$$

Furthermore,

$$
\text { pTime }_{i} \leq 2 \cdot \text { bounceSteps }_{i}+1+\text { returnSteps }_{i}+\text { forwdSteps }_{i}
$$

Where, $2 \cdot$ bounceSteps $_{i}+1$ is an upper bound on the time needed by agent $B$ to switch state from Bounce to Return given by the predicate "Etime $>2$ Esteps". The quantity returnSteps $_{i}+$ forwdSteps $_{i}$ is an upper bound on the time needed for $B$ to catch again agent $F$. Substituting returnSteps into the latter yields

$$
\text { pime }_{i} \leq 3 \cdot \text { bounceSteps }_{i}+2 \cdot \text { forwdSteps }_{i}+1
$$

If the agents do not terminate after this catch, it must be forwdSteps $s_{i}>$ bounceSteps $_{i}$, hence $p$ Time $_{i} \leq$ 6 - forwdSteps $s_{i}$. This means that by time $12 n$ at the latest since the first catch, agent $F$ will know $n$ and will terminate in $7 n$ further rounds (if it does not terminate earlier due to some other terminating condition). The correctness now follows from Lemma 2 and optimality is obvious. 
States: $\{$ InitL, Happy, FirstBlockL, AtLandmarkL, Ready, Reverse, Bounce, Return, Forward, Terminate, BComm, FComm \}. In state InitL:

$\operatorname{dir} \leftarrow$ left $, k_{1} \leftarrow 0, k_{2} \leftarrow 0, k_{3} \leftarrow 0$

LEXPLORE( $\operatorname{dir} \mid n$ is known: Happy; Btime $>0$ : FirstBlockL; catches: Bounce; caught: Forward)

In state Happy:

LEXPLORE $($ dir $\mid$ Ttime $\geq 32((3\lceil\log (n)\rceil+3) 5 \cdot n)+1$ : Terminate; catches: Bounce; caught: Forward $)$

In state FirstBlockL:

dir $\leftarrow$ right,$k_{1} \leftarrow$ Ttime -1

LEXPLORE $(\operatorname{dir} \mid n$ is known: Happy, isLandmark: AtLandmarkL; Btime > 0: Ready; catches: Bounce; caught: Forward)

In state AtLandmarkL:

$k_{3} \leftarrow$ Etime

if both agents are at the landmark then

Wait one round

if both agents are at the landmark then

Terminate

LExplore(dir $\mid n$ is known: Happy, Btime > 0: Ready; catches: Bounce; caught: Forward)

In state Ready:

$k_{2} \leftarrow$ Etime

Compute your ID by interleaving bits of the bit-strings representation of $k_{1}, k_{2}$ and $k_{3}$. Each $k_{i}$ string of bits is padded by a prefix 0 until its length is equal to the biggest of the three. $\operatorname{set}(\mathrm{ID})$

Change to state Reverse and process it

In state Reverse:

dir $\leftarrow$ direction (Ttime)

if $n$ is known then

LEXPLORE $(\operatorname{dir} \mid$ Ttime $\geq 32((3\lceil\log (n)\rceil+3) 5 \cdot n)$ : Terminate; catches: Bounce; caught: Forward $)$ else

LEXPLORE(dir | switch(Ttime): Reverse; catches: Bounce; caught: Forward)

In state Bounce, Return, Forward, BComm, FComm:

The same as in Algorithm LandmarkWithChirality.

Figure 8: Algorithm StartFromLandmarkNoChirality 


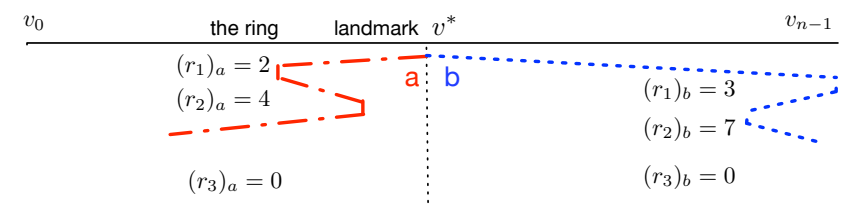

$$
\begin{gathered}
\text { Bitstrings and ID of a } \\
\begin{array}{c}
\left(k_{1}\right)_{a}=010 \quad\left(k_{2}\right)_{a}=010 \\
\left(k_{3}\right)_{a}=000 \\
(I D)_{a}=110000=(48)_{10}
\end{array}
\end{gathered}
$$

$$
\begin{gathered}
\text { Bitstrings and ID of b } \\
\left(k_{1}\right)_{b}=011 \quad\left(k_{2}\right)_{b}=100 \\
\left(k_{3}\right)_{b}=000 \\
(I D)_{b}=010100100=(164)_{10}
\end{gathered}
$$

Figure 9: Example of a run where different IDs are assigned to agents. In this case the round $r_{3}$ is 0 for both agents; no one visits the landmark between $r_{1}$ and $r_{2}$.

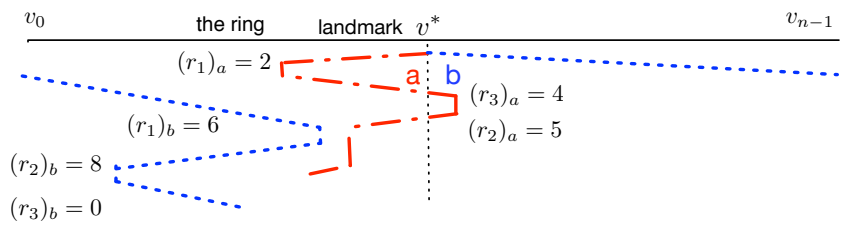

$$
\begin{gathered}
\text { Bitstrings and ID of a } \\
\left(k_{1}\right)_{a}=10 \quad\left(k_{2}\right)_{a}=01 \\
\left(k_{3}\right)_{a}=10 \\
(I D)_{a}=101010=(42)_{10}
\end{gathered}
$$

$$
\begin{gathered}
\text { Bitstrings and ID of } \mathrm{b} \\
\left(k_{1}\right)_{b}=110 \quad\left(k_{2}\right)_{b}=010 \\
\left(k_{3}\right)_{b}=000 \\
(I D)_{b}=100110000=(304)_{10}
\end{gathered}
$$

Figure 10: Example of a run where different IDs are assigned to agents. In this case $r_{3} \neq 0$ for agent $a$.

\subsubsection{Termination: Landmark without Chirality}

In this subsection we consider the case of a landmark when there is no chirality, and we prove that exploration with termination can still be performed in time $O(n \log n)$.

We first consider and solve the problem when both agents start from the landmark; we then adapt the algorithm to work when agents start in arbitrary positions.

Starting from the Landmark. The pseudocode of Algorithm StarTFromLandmarkNoChirality is in Figure 8. The main difficulty lies in the case when the agents start in opposite directions and never break the symmetry. Our approach to solve this case is to add an initial phase in which the agents use the event of waiting on a missing edge to break symmetry, obtain different IDs (of size $O(\log n)$ ) and then use these IDs to ensure that, if the agents do not catch each other (or outright explore the ring), then they eventually move in the same direction for a sufficiently long time so that Algorithm LANDMARKWITHCHIRALITY succeeds.

Let us remark that, if the agents somehow catch each other, they establish chirality, and then they can use Algorithm LANDMARKWithChirality which leads to exploration and termination. Therefore, if at any point the agents catch each other, they enter states Forward and Bounce and proceed with Algorithm LANDMARKWITHCHIRALITY.

Computing the ID: Each agent tries to compute its ID according to the procedure described below. If an agent does not succeed in computing its ID, then it has explored the ring and it is aware of that.

If an agent does not know the ring size, the first two times it waits in a port it immediately changes direction. We indicate these rounds with $r_{1}$ and $r_{2}$, respectively. Let $r_{3}$ be the round when the agent entered the landmark for the first time between times $r_{1}$ and $r_{2}\left(r_{3}\right.$ is set to 0 if the agent does not traverse the landmark between rounds $r_{1}$ and $\left.r_{2}\right)$. Let $k_{2}=\left(r_{2}-\max \left(r_{1}, r_{3}\right)\right)$ and $k_{3}=\max \left(0,\left(r_{3}-r_{1}\right)\right)$; note that 


\begin{tabular}{|c|c|c|c|c|}
\hline Phases: & 1 & 2 & 3 & 4 \\
\hline Direction: & \begin{tabular}{|l|l:l}
0 & 0 & 0 \\
\end{tabular} & \begin{tabular}{|l:l:l:l|l|l|}
1 & 0 & 1 & 0 & 1 & 1 \\
\end{tabular} & 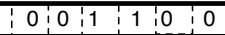 & $\ldots \ldots$ \\
\hline
\end{tabular}

Figure 11: Directions for an agent with $I D=1$, a round with value $0 / 1$ corresponds to left/right direction.

these values are provided by variable Etime in the algorithm (see Figure 8). The computed ID of this agent consists of the interleaved bits of the numbers $k_{1}=r_{1}, k_{2}$ and $k_{3}$, where each bitstring is padded with a prefix of 0 's until its length is equal to that of the longest of the three. Note that two IDs are equal if and only if their $k_{i}$ 's are equal. In Figure 9 there is a detailed example on how the IDs are computed; notice that since both IDs start with 0 , this bit is ignored when the numerical value in base 10 is obtained. In Figure 10 there is an example of a run where $r_{3} \neq 0$ for agent $a$.

Moreover, notice that if a round $r_{1}$ or $r_{2}$ does not exist, because the agent encountered a missing edge less than two times, then that agent has looped around the landmark; in this case it enters in the Happy State (cf. pseudocode). So it knows the ring size and it can compute an upper bound on the termination time of the other agent.

Using the IDs to decide the direction: The following procedure is used when an agent has computed its ID. Agents agree on a predetermined subdivision of rounds in phases. Round $r$ belongs to phase $j, r \in$ phase $(j)$, iff $2^{j} \leq r<2^{j+1}$. Given the ID, an agent computes a string of bits $S(I D)=10 \circ(b(\mathrm{ID})) \circ 0$, where $\circ$ is the string concatenation and $b$ (ID) is the minimal binary representation of ID. Given a string $S$ we use $(S)_{i}$ to denote the $i-t h$ character of $S$. Let us define as $\bar{j}$ the minimum value for which $2^{\bar{j}} \geq \operatorname{len}(S(I D))$ and $\overline{S(I D)}=(0)^{2^{\bar{j}}-\operatorname{len}(S(I D))} \circ S(I D)$, where $\operatorname{len}(S)$ is the length of the string $S$. For each phase $j \geq \bar{j}$ we associate the binary string $d(I D, j)=\operatorname{Dup}\left(\overline{S(I D)}, 2^{(j-\bar{j})}\right)$, where $\operatorname{Dup}(S, k)$ is the string obtained from $S$ by repeating each character $k$ times, e.g. $\operatorname{Dup}(1010,2)=11001100$.

For each round $r \in$ phase $(j)$, with $j>\bar{j}$, the direction of the agent is equal to left if $(d(I D, j))_{r-2^{j}}=0$, otherwise it is right. For a round $r \in$ phase $(j)$ with $j \leq \bar{j}$ the direction of an agent is fixed to left.

In Figure 11 there is an example of the bit sequences generated by an agent with $I D=1$.

In our algorithm this procedure is implemented using three functions:

- $\operatorname{set}(I D)$ : This function takes as parameter the ID of the agent, and it initializes the aforementioned procedure.

- direction(Ttime): This function takes as parameter the current round and it returns the direction according to the aforementioned procedure.

- $\operatorname{switch}($ Ttime): This function takes as parameter the current round and it returns true if direction(Ttime) $\neq \operatorname{direction}($ Ttime -1$)$.

Lemma 3. Let us consider two agents with different IDs: $\left\{I D, I D^{\prime}\right\}$, with len $(I D) \geq \operatorname{len}\left(I D^{\prime}\right)$. For any constant $c>0$, by round $r<32(($ len $(I D)+3) c \cdot n)+1$ there has been a sequence of $c \cdot n$ rounds in which the agents had the same direction. Moreover, by round $r$, each agent has moved in both directions for a sequence of rounds of length at least $c \cdot n$.

Proof. By definition $\bar{j}=\lceil\log (\operatorname{len}(I D)+3)\rceil$. Phase $\bar{j}$ starts at round $r<2^{\log (\operatorname{len}(I D)+3)+2}<4(\operatorname{len}(I D)+3)$. Consider $d(I D, \bar{j})$ and $d\left(I D^{\prime}, \bar{j}\right)$. We now show that there exist two indices $x, y$ such that $(d(I D, \bar{j}))_{x}$ $\neq\left(d\left(I D^{\prime}, \bar{j}\right)\right)_{x}$ and $(d(I D, \bar{j}))_{y}=\left(d\left(I D^{\prime}, \bar{j}\right)\right)_{y}$. It is easy to verify that $y=\operatorname{len}(d(I D, \bar{j}))$, by construction since $d($.$) always terminate with 0$. For the index $x$ we consider two cases:

- $d\left(I D^{\prime}, \bar{j}\right)=\overline{S\left(I D^{\prime}\right)}$ : If $\operatorname{len}(S(I D))>\operatorname{len}\left(S\left(I D^{\prime}\right)\right)$ then $x$ is the index of the first bit of $d_{s}(I D, \bar{j})$ different from zero. If $\operatorname{len}(S(I D))=\operatorname{len}\left(S\left(I D^{\prime}\right)\right)$, since by assumption $I D \neq I D^{\prime}$ index $x$ exists. 


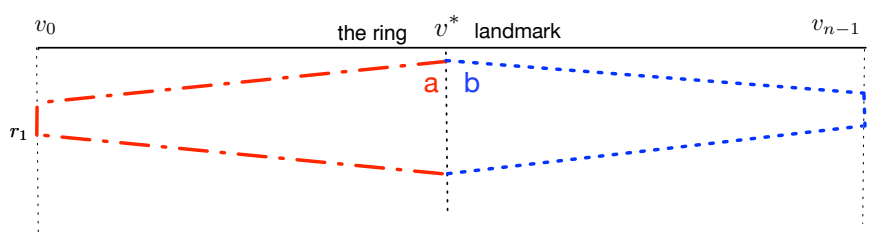

Figure 12: Termination from state AtLandmark.

- $d\left(I D^{\prime}, \bar{j}\right) \neq \overline{S\left(I D^{\prime}\right)}$ : By construction, $d_{s}\left(I D^{\prime}, \bar{j}\right)$ is composed by sequences of equal bits of length at least two. Also by construction, the first three bits of $S(I D)$ are 101. This means that the substring 101 cannot be contained in $d\left(I D^{\prime}, \bar{j}\right)$, and it is contained in $d(I D, \bar{j})$; this implies that index $x$ exists.

If the agents agree on the direction, they will have the same direction in the round corresponding to index $y$, otherwise they will have the same direction in the round corresponding to index $x$.

In phase $j>\bar{j}$, by construction, we have a sequence of rounds where agents have the same direction of length at least $2^{j-\bar{j}}$. We have $2^{j-\bar{j}}>c \cdot n$ when $j>\log (\operatorname{len}(I D)+3)+\log (c \cdot n)+3$. We reach the end round of this phase by $r=\sum_{i=0}^{\log (\operatorname{len}(I D)+3)+\log (c \cdot n)+4} 2^{i} \leq 32((\operatorname{len}(I D)+3) c \cdot n)$. The last statement of the lemma derives directly by the presence of 1 and 0 in each possible $S(I D)$.

Theorem 7. Algorithm STARTFromLANDMARKNoCHIRALITY allows two anonymous agents without chirality starting from the landmark to explore a 1-interval connected ring with a landmark and to explicitly terminate in $O(n \log (n))$ time.

Proof. First note that if the agents catch each other, as shown in the proof of Theorem 6, they will explore the ring and terminate in $O(n)$ time after the moment they catch; hence, in the remainder of the proof, we deal with the case when the agents never catch each other. Next note that, if the agents meet at the landmark and terminate in state AtLandmark, they must have bounced from the same edge and the ring has been explored; this is because they started from the landmark and returned at the same time while both were blocked exactly once; see Figure 12. However, when two agents meet in the landmark and one terminates, to ensure that the other is in state AtLandmark the following synchronization step is needed: when an agent enters state AtLandmark and sees also the other agent, it waits one round more in the node without moving. If the other agent does the same they both terminate. This obviously happens in the aforementioned case, i.e. when the agents bounced on the same edge and reach the landmark at the same time, entering both in the same round in state AtLandmark, thus correctly identifying the exploration of the ring. The same cannot happen if one of the agents is not in the AtLandmark state; in this case only one of the agent will wait and the other will either leave the node, or it will enter a port, thus preventing a possible incorrect termination.

Third, observe that, by time $3 n-1$, either an agent knows $n$ (and terminates in $O(n \log (n)$ ) time from Happy state, or it knows its own ID. Note that IDs are bounded from above by $n^{3}$, since each $k_{i}$ is at most $n$, which implies $\operatorname{len}(I D) \leq 3\lceil\log (n)\rceil$.

Consider now the case that at time $3 n-1$ an agent (say a) does not know its ID (and hence since time $3 n-1$ knows $n$ ), while the other (b) knows its ID but does not know $n$. Agent $b$ therefore repeatedly switches its direction in state Reverse, while agent $a$ moves in the same direction. Note that by Lemma 3 , by time $32((3\lceil\log (n)\rceil+3) 5 \cdot n)+1$, agent $b$ has moved to the left and right direction for a sequence of rounds of length at least $5 n$, in one of the two both $a$ and $b$ move in the same direction. As at least one agent makes progress in each of those time steps, while (by assumption) they don't catch each other, $b$ must have moved for at least $2 n$ time units. This means that $b$ learns $n$ and eventually terminates as well.

The final case to consider is when both agents know their IDs, but do not know $n$. Note that if the agents have the same values of $k_{1}$ and $k_{2}$, they must have covered the whole ring and at least one of them will have $k_{3} \neq 0$. This means that the agents necessarily have different IDs, since if they had the same values of $k_{1}$ and $k_{3} \neq 0$, they would have terminated in AtLandmark state, see Figure 12. 
States: $\{$ Init, AtLandmark, InitL, FirstBlock, FirstBlockL, AtLandmarkL, Ready, Reverse, Bounce, Return, Forward, Terminate, BComm, FComm .

In state Init:

$\operatorname{dir} \leftarrow$ left $, k_{1} \leftarrow 0, k_{2} \leftarrow 0, k_{3} \leftarrow 0$

LEXPLORE(dir $\mid n$ is known: Happy; Btime $>0$ : FirstBlock; catches: Bounce; caught: Forward;)

In state FirstBlock:

dir $\leftarrow$ right,$k_{1} \leftarrow$ Ttime

LEXPLORE(dir $\mid n$ is known: Happy; isLandmark: AtLandmark; Btime > 0: Ready; catches: Bounce; caught: Forward)

In state AtLandmark:

$k_{3} \leftarrow$ Etime

if both agents are at the landmark then

Wait one round

if both agents are at the landmark then go to state InitL

LEXPlORE(dir $\mid n$ is known: Happy; Btime $>0$ : Ready; catches: Bounce; caught: Forward)

In state $S \notin\{$ Init, FirstBlock, AtLandmark $\}$ :

The same as in Algorithm StartAtLandmarkNoChirality.

Figure 13: Algorithm LandmarkNoChirality

Since the IDs are different, by Lemma 3 , by round $32((3\lceil\log (n)\rceil+3) 5 \cdot n)+1$ there has been a time segment of length $5 n$ in which both agents were moving in the same direction. Thus, either they catch each other, or both learnt $n$ and terminated thereafter.

Arbitrary initial positions. Algorithm StartAtLandmarkNoChirality almost works also in the case of agents starting in arbitrary position. The only failure would be due to the fact that, when the agents meet in the landmark while establishing $k_{1}$ and $k_{2}$, it does not necessarily mean that they have already explored the ring. The modification to introduce is not to terminate in this case, but to reset and start a new instance in state InitL, executing algorithm StartAtLANDMARKNoChirality, as now the agents are indeed starting at the landmark. If the agents do not meet at the landmark, then their values of $k_{3}$ are different and the algorithm works using the same arguments. The complete pseudocode is in Figure 13 Since this adds at most $O(n)$ to the overall time, we obtain the following theorem.

Theorem 8. Algorithm LANDMARKNoChiRALity allows two anonymous agents without chirality to explore a 1-interval connected ring with a landmark and to explicitly terminate in $O(n \log (n))$ time.

\section{Ring exploration in $\mathcal{S S Y \mathcal { N C }}$}

In this section we investigate the exploration problem when the system is semi-synchronous. The complexity measure we consider in this case is the total number of edges traversed by the agents. As in Section 3 , Esteps denotes the total number of successful moves performed by the agent since procedure EXPLORE has been called, Tnodes denote the total number of nodes that the agent perceived to have explored since the beginning of the protocol, Btime denotes the number of consecutive rounds the agent has been currently waiting in a port, and catches is the predicate denoting that the agent is in the node and the other agent is observed on a port (in the moving direction of the first agent). These definitions hold for all the algorithms of the $\mathcal{S} \mathcal{S Y N C}$ section.

\subsection{Impossibility of Exploration in NS}

Let us begin by showing an intuitive result for the NS model: 
Theorem 9. In the NS model, exploring the ring is impossible with any number of agents, even if the ring and the agents are not anonymous and there is chirality.

Proof. Consider a non-anonymous ring where $k>1$ non-anonymous agents are located at arbitrary nodes, with at least a node without agents; and let $\mathcal{A}$ be an arbitrary exploration algorithm. Starting with $t=0$, let $A(t)$ denote the set of agents that, according to $\mathcal{A}$, if active at time $t$ would want to move to a neighbouring node; let $P(t)$ denote the set of agents that instead would not move; and let $f i r s t(t) \in A(t)$ be the agent in $A(t)$ that has not been active the longest, where ties are arbitrarily broken.

Consider now the following agents and link activation scheduler: at time $t$ it activates only $P(t)$ and first $(t)$, and it removes the edge on which $f$ irst $(t)$ would move. Hence, no agent will move at time $t$. By repeating this process and by observing that this scheduler is indeed fair (it activates every agent infinitey often), the nodes initially without an agent will never be visited.

Notice that Theorem 9 would hold even if the agents were equipped with wireless communication.

Motivated by this impossibility result, we now examine the other $\mathcal{S} \mathcal{S} \mathcal{N C}$ models.

\subsection{Exploration in PT}

\subsubsection{PT: Impossibility of Exploration by Two Agents Without Chirality}

We begin our investigation of the PT Model by showing that, without chirality, two agents cannot explore the ring, even with precise knowledge of the network size and with the presence of a landmark.

Theorem 10. In the PT model without chirality two anonymous agents are not sufficient to explore a ring of size $n \geq 5$. The result holds even if there is a distinguished landmark node and the exact network size is known to the agents.

Proof. By contradiction, let $\mathcal{A}$ be a solution algorithm. Let $a$ and $b$ be the two agents. Assume that: agent $a$ starts at node $u$ and, according to $\mathcal{A}$, it would move towards $u^{\prime} ; b$ starts at node $v$ and would move towards $v^{\prime}$; and $u, u^{\prime}, v, v^{\prime}$ and the landmark are all different.

The algorithm is executed by the agents against an adversary that choses which agent is active in which round, decides the local orientation of the agents, as well as the topological structure of the ring. In particular, it does not fix the link relationship between the nodes where $a$ acts $\left(u, u^{\prime}\right)$ and those where $b$ operates $\left(v, v^{\prime}\right)$, until necessary during the execution of the algorithm $\mathcal{A}$.

The adversary applies the following alternation strategy: It activates only $a$ and keeps it active until $a$ tries to move to a node other than $u$ and $u^{\prime}$. At this point, the adversary blocks that edge and keeps it blocked until $a$ either switches direction, or decides to permanently wait on this port at node $u^{*} \in\left\{u, u^{\prime}\right\}$. Notice that one of these two events has to take place. When this happens, the adversary makes $a$ passive, activates $b$, and acts with $b$ exactly in the same way as it did with agent $a$. In other words, it keeps $b$ active until $b$ tries to move to a node other than $v$ and $v^{\prime}$ and then it blocks that edge and keeps it blocked until $b$ either switches direction, or decides to permanently wait on this port at node $v^{*} \in\left\{v, v^{\prime}\right\}$.

Note that, after the execution of the alternation strategy, since the agents are anonymous and there is no chirality, whatever decision $a$ made (switch or permanently wait), the same is taken by agent $b$.

Let us consider first the case when both agents decide to switch direction. In this case, the adversary will continue, as long as the two agents decide to switch direction, to execute the alternating strategy, never letting the agents move outside the four nodes $u, u^{\prime}, v, v^{\prime}$. This means that, if they never decide to wait permanently, the rest of the ring will not be explored, contradicting the correctness of $\mathcal{A}$.

Therefore, within finite time, after some executions of the alternating strategy, they both must decide to wait permanently, $a$ at node $u^{*}$ to go to node $u^{\prime \prime}$, and $b$ at node $v^{*}$ to go to node $v^{\prime \prime}$. When this happens, the adversary fixes the topology of the ring by setting $u^{\prime \prime}=v^{*}$ and $v^{\prime \prime}=u^{*}$; it then blocks forever edge $\left(u^{*}, v^{*}\right)$, and permanently activates both $a$ and $b$. Thus the rest of the ring will not be explored, contradicting the correctness of $\mathcal{A}$. 
As a consequence of Theorem 10, any exploration algorithm must either use chirality, or employ more than two agents. We will consider the two cases in the following subsections.

\subsubsection{PT: Two Agents with Chirality}

We know (Theorem 10 that two agents need chirality to explore the ring; however chirality does not suffice for exploration with termination (Theorem 1) unless the agents know an upper bound on the ring size or the nodes are not anonymous (i.e., there is a landmark).

Interestingly, in the PT model, even with both knowledge of the the ring size and a landmark, explicit termination of both agents is impossible as shown by the following theorem.

Theorem 11. In the PT model, any exploration algorithm on a ring of size $n \geq 3$ with two agents can only guarantee partial termination. The result holds even if the size of the ring is known, a landmark node is present and there is chirality.

Proof. By contradiction, let $\mathcal{A}$ be a two-agents exploration algorithm for PT, where both agents always terminate in every execution. Let $a, b$ be the two agents, and let them start at the same location, the landmark node $v_{s}$, both initially asleep. First notice that, if only one agent wakes up and the other remains asleep, eventually the awake agent has to start exploring the ring (otherwise, by alternating the sleeping of $a$ and $b$, they will never move).

Let $E(a)$ (resp. $E(b))$ be the unfair execution of $\mathcal{A}$ in which $a$ (resp. $b$ ) forever sleeps, $b$ (resp. $a$ ) is active and explores the ring, and no edge is removed. Observe that, since the agents are anonymous, then $a$ does in $E(b)$ the same moves as $b$ does in $E(a)$.

Let us now examine the four possible situations in which $b$ can find itself in $E(a)$ :

1. Agent $b$ terminates in some location $v_{x} \neq v_{s}$. Consider now the execution $E^{\prime}$ coincident with $E(a)$ until the round when $b$ terminates in $v_{x}$; then the adversary wakes up $a$ and blocks it on $v_{s}$; hence $a$ will never leave $v_{s}$. Observe that $E^{\prime}$ is a fair execution; since, by assumption, both agents explicitly terminate in every fair execution, then $a$ will terminate in $E^{\prime}$ on node $v_{s}$, say $t$ rounds after becoming awake.

Consider now an execution $E^{\prime \prime}$ coincident with $E(a)$ until $b$ moves to a node $v_{y} \neq v_{s}$; when this happens $a$ is woken up and blocked on node $v_{s}$, while $b$ is kept asleep. From the local view of $a$, the executions $E^{\prime}$ and $E^{\prime \prime}$ are not distinguishable; hence $a$ terminates in $E^{\prime \prime}$ on $v_{s} t$ rounds after waking up. When this happens, the adversary wakes up $b$ and blocks it on node $v_{y}$. Observe that also $E^{\prime \prime}$ is a fair execution. As $n \geq 3$ and only $v_{s}$ and $v_{y}$ are visited in this execution, the ring will never be explored, contradicting the correctness of $\mathcal{A}$.

2. Agent $b$ terminates on node $v_{s}$. Consider now the execution $E^{\prime}$ coincident with $E(a)$ until the round when $b$ terminates in $v_{s}$; when this happens the adversary wakes up $a$. Since $a$ does not know that $b$ has terminated and $b$ 's behaviour is indistinguishable from being asleep, $a$ has to leave $v_{s}$. When it reaches a neighbouring node $v_{y}$, the adversary blocks it there. Notice that $E^{\prime}$ is a fair execution. Consider next the execution $E^{\prime \prime}$ coincident with $E(b)$ (i.e., $a$ is made active while $b$ is asleep) until $a$ leaves $v_{s}$; when it reaches a neighbouring node $v_{y}$, also in this case the adversary blocks it there.

Observe that $a$ cannot distinguish between executions $E^{\prime}$ and $E^{\prime \prime}$; hence $a$ will take the same decisions in both. If it terminates within finite time, then, when that happens, in execution $E^{\prime \prime}$ the adversary awakes $b$ and perpetually blocks it on $v_{s}$; hence the ring will never be explored. Since $E^{\prime \prime}$ is a fair execution, this contradicts the correctness of $\mathcal{A}$. If, on the other hand, a does not terminate, execution $E^{\prime}$ contradicts the assumption that $\mathcal{A}$ always guarantees explicit termination.

3. Agent $b$ does not terminate, and it visits $v_{s}$ only finitely many times. Hence, there is a round $r$ after which $b$ does not visit $v_{s}$ ( $b$ can be waiting at some node $v_{x} \neq v_{s}$; it can also be perpetually moving among a set of nodes not containing $v_{s}$ ). 


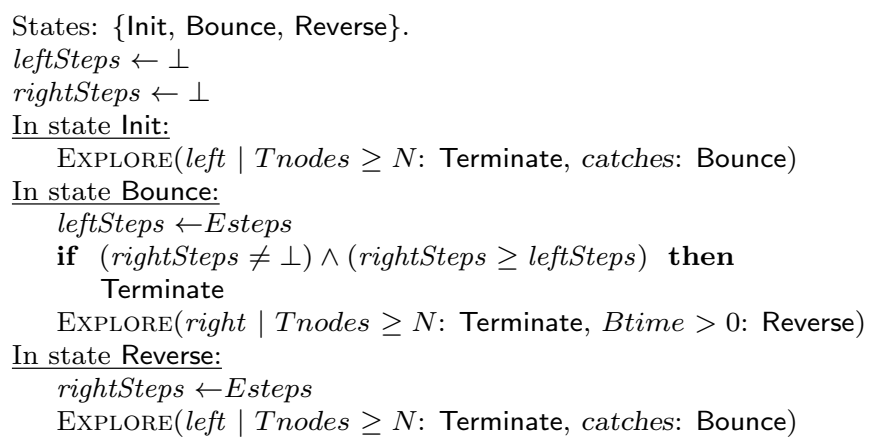

Figure 14: Algorithm PTBoundWithChiRALity

Consider now the execution $E^{\prime}$ coincident with $E(a)$ for the first $r$ rounds; the adversary then wakes $a$ at round $r+1$ and blocks it on $v_{s}$ forever; notice that $E^{\prime}$ is fair. By assumption, both agents terminate in $\mathcal{A}$. In particular, this means that $a$ terminates on $E^{\prime}$ without leaving $v_{s}$.

Consider now an execution $E^{\prime \prime}$ coincident with $E^{\prime}$ until the first time $b$ visits a node $v_{y} \neq v_{s}$. From that moment on, $b$ becomes asleep in $v_{y}$, while $a$ is activated and blocked on $v_{s}$. From the point of view of $a$ this execution is undistinguishable from $E^{\prime}$, therefore $a$ terminates in $v_{s}$. After the termination of $a$, the adversary wakes up $b$ in $E^{\prime \prime}$ but perpetually blocks it; notice that $E^{\prime \prime}$ is fair. As only $v_{s}$ and $v_{y}$ have been explored in this execution, $\mathcal{A}$ fails to explore the ring.

4. Agent $b$ does not terminate, and it visits $v_{s}$ infinitely often, either by reaching it and no longer moving or by moving inside an interval $I$ of nodes containing $v_{s}$. Consider now the execution $E^{\prime}$ coincident with $E(a)$ until $b$ returns to $v_{s}$; the adversary then puts $b$ to sleep and wakes up $a$ until it returns to $v_{s}$. Now, the adversary will repeate the whole process, alternating forever the sleep of $a$ and $b$ : wake up $b$ (resp. $a$ ) and put $a$ (resp. $b$ ) to sleep, util the next round in which $b$ (resp. $a$ ) is at $v_{s}$ (recall that it might never leave it). Notice that $b$ is unable to distinguish this fair execution $E$ from $E(a)$, and similarly $a$ is unable to distinguish it from $E(b)$; hence they will never terminate in this execution.

As a consequence, the best that can be achieved is partial termination.

In the rest of this section, we show that the knowledge of an upper bound or the presence of a landmark is sufficient for the two agents to explore the ring; with respect to termination, we achieve a strong partial termination, with one agent always explicitly terminating, and the other either terminating or no longer moving.

\section{A. Chirality and Known Upper Bound}

First consider the case when an upper bound $N$ on the ring size is known to the agents. We present an algorithm, PTBoundWithChirality, shown in Figure 14, for exploration of dynamic ring by two agents with chirality and knowledge of an upper bound. Both agents start moving left. If an agent finds a blocked edge with the other agent waiting in the left port, it enters state Bounce, reverses direction and starts moving right. If the agent in state Bounce finds a missing edge before traversing $N$ edges, it reverses direction again (becoming Reverse); that agent might be alternating Bounce and Reverse state several times. An agent terminates upon discovering it has traversed $N$ consecutive edges in a given direction. Additionally, if in state Reverse the agent catches the other agent at a distance smaller than that in the previous catch, it means the two agents have crossed and they can safely terminate. 


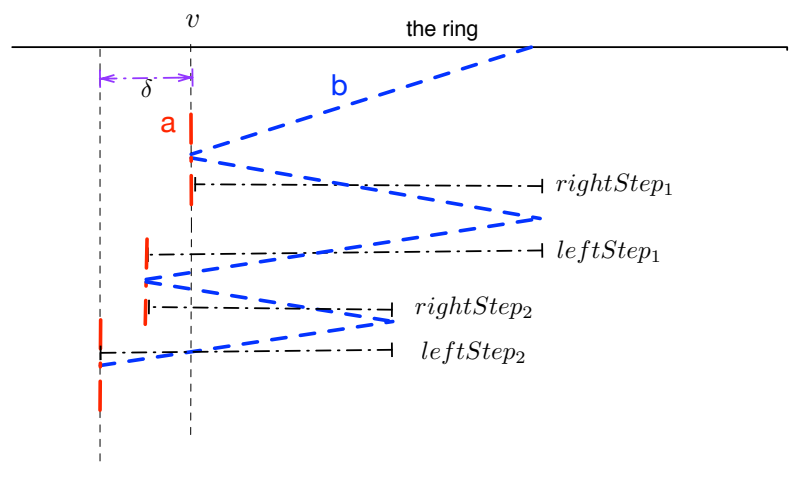

Figure 15: Run where rightSteps < leftSteps, the $\delta$ grows at each bounce-reverse of $b$.

Theorem 12. Two agents executing Algorithm

PTBoundWITHChIRALITY in the PT model with chirality and a known upper bound $N$ on the size of the ring will explore the ring using at most $O\left(N^{2}\right)$ edge traversals. Furthermore, one agent explicitly terminates, while the other either terminates or it waits perpetually on a port.

Proof. We first prove exploration. Note that, by definition of Tnodes, if it exceeds $N$, the ring has been explored. The only non-trivial case to consider is termination due to the condition: rightSteps $\geq$ leftSteps.

Let us first consider the case when where agent $b$ terminates after it bounces on agent $a$ blocked on edge $e$ at round $r$, then changes direction on a missing edge and finally catches $a$ again terminating.

If $a$ stayed in the same port during all this process, then $b$ would have visited the other side of $e$ (otherwise the PT condition would have ensured passive transport of $a$ ). In such a case the ring has been explored.

If $a$ moved after round $r$, two different scenarios are possible:

- (i) $a$ crosses $b$ while $b$ is in Bounce state, this implies that $a$ and $b$ explored the ring.

- (ii) $a$ bounced on $b$ while $b$ was in Reverse, $a$ traverses $e$ in state Bounce, and $a$ goes back in state Reverse. It is clear that also in this case the ring has been explored: the portion between $e$ and the node where $a$ bounced on $b$ has been explored by $b$, and the other portion by $a$.

We now have to show that at least one agent terminates. If the adversary keeps an edge perpetually removed, eventually the algorithm terminates due to condition rightSteps $=$ leftSteps. Moreover, if an agent is not blocked in its traversal, it will eventually do $N$ steps leading to termination.

The only possibility left to be analyzed is if $a$ is blocked on some edge $e, b$ bounces first on edge $e$, then reverses direction on edge $e^{\prime}$ and, when $b$ catches on $a$, rightSteps < leftSteps. Notice that, when this happens, $b$ has done at least one step further to the left of edge $e$, otherwise we would have rightSteps $\geq$ leftSteps leading to the termination of $b$.

We have that (1) the adversary cannot keep an agent sleeping, or blocked, forever, (2) the adversary cannot let an agent do $N$ steps in one direction and (3) an agent switches from state Reverse to Bounce only catching someone; therefore if the agents do not terminate, then $a$ and $b$ have to catch each other multiple times. As discussed previously, for each catch the catching agent will do an additional step to the left. Therefore, we will eventually have Tnodes $>N$ for one of the two. An example is reported in Figure 15 , in the figure we can see that after the first bounce on node $v$, the area to the left of $v$ explored by $b$ (named $\delta$ in the figure) has to grow at least by one for each sequence of Reverse-Bounce: we must have rightSteps $s_{i}<$ leftStep $_{i}$.

If an agent terminates, the other cannot bounce to the right. Hence, it will either terminate due to exceeding $N$ left moves, or will be perpetually blocked on a port, and the last part of the theorem holds.

Let us now analyze the complexity of the algorithm. Observe that during one Bounce-Reverse phase an agent can do $O(N)$ steps. There could be at most $N$ of these Bounce-Reverse phases: in each of them the 
agent has to do an additional step left otherwise the termination condition is satisfied. Since the termination check bounds the total number of left steps by $N$, this yields $O\left(N^{2}\right)$ complexity of the algorithm.

The complexity of the proposed algorithm, $O\left(N^{2}\right)$, depends on the accuracy of the upper bound $N$. We will now show that such a dependency is to a certain degree inevitable. In fact we prove that $\Omega(N \cdot n)$ are indeed required by any solution protocol. This also means that the proposed algorithm is optimal, whenever $N=O(n)$.

Theorem 13. In the PT model with chirality in which the two agents know an upper bound $N$ on the ring size $n \geq 5$, any exploration algorithm with partial termination requires at least $\Omega(N \cdot n)$ edge traversals by the agents in the worst case.

Proof. Let $\mathcal{A}$ be a solution algorithm for the PT model with chirality and let $N$ be the known upper bound on the ring size. The actual size $n \leq N$ of the ring will be decided by the adversary. The adversary will operate in logical phases; in phase $i$, it selects a continuous segment $X_{i}$ of the ring that includes both agents; initially $X_{1}$ has size $x \geq 5$. In each phase, the adversary uses the strategy described below. Let $N_{i}$ denote the number of distinct nodes that the agents would have explored, under this strategy, at the beginning of phase $i$ if $n=N$, i.e. the upper bound was tight. We assume, without loss of generality, that $N_{1}=\left|X_{1}\right|=x$; this only makes the lower bound proof stronger. The adversary repeats the same process as long as $N_{i}<N$.

In phase $i$ the adversary considers one agent at a time. If that agent, say $a$, tries to leave $X_{i}$, the adversary will block that edge and keeps it blocked until either $a$ changes direction (i.e., stays within $X_{i}$ ) or also the other agent tries to leave $X_{i}$ (from opposite directions).

Eventually both agents must want to leave $X_{i}$ from opposite directions, otherwise the adversary can continue to prevent the agents from leaving $X_{i}$ and since $N_{i}<N$, choosing $n>N_{i}$ would make the algorithm $\mathcal{A}$ fail.

When the adversary determines that also the second agent, say $b$, would want to leave $X_{i}$ should it become active (recall the first agent is currently waiting on a blocked edge), the adversary considers what each agent would do, according to $\mathcal{A}$, if the edge the agent wants to cross is blocked indefinitely.

Clearly, they cannot both wait indefinitely, otherwise the adversary, by choosing $n=N_{i}$ and activating both agents at all times, would make the two agents wait forever at the two endpoints of the same blocked edge, without either of them terminating (although they have explored the ring).

This means that at least one of the agents, after a finite number of activations, would change direction and move towards a neighbouring node in $X_{i}$. Let $c \in\{a, b\}$ be such an agent, and $d$ be the other. Upon this determination, if $d=b$, the adversary activates $d$ (letting it move), and then makes $d$ passive; otherwise, i.e. $d=a$, it makes $d$ passive immediately, and let it move passively on the next node. In any case, it also blocks the edge from which $c$ is trying to leave $X_{i}$ until $c$ changes direction and moves from its current position $u$ to the next node $u^{\prime}$ in $X_{i}$. At this point, the adversary blocks the edge $\left(u, u^{\prime}\right)$ and keeps it blocked until $c$ performs at least $\left|X_{i}\right|-1$ additional moves, possibly reaching $d$. Note that this will always happen as shown by the claim below.

Claim 1. If c never reaches $d$ in this phase and it does not wait forever in the port of node $u^{\prime}$ trying to reach node $u$, it will perform an unbounded number of moves.

Proof. By contradiction, let $c$ satisfy the conditions of the claim, but perform only a bounded number of moves, without reaching $d$. Thus, within finite time it will stop in a node indefinitely. When this happens, the adversary activates $d$ blocking any edge through which it wants to move. Hence no new node will be explored; since $N_{i}<N$, by choosing $n=N$ the adversary would make the algorithm $\mathcal{A}$ fail.

Further note that, during this time, agent $d$ moves (if $d=b$ ) or is passively moved (if $d=a$ ) to a neighbour $v$ outside $X_{i}$.

In Claim 1 we assumed that $c$ does not wait forever in the port of node $u^{\prime}$ trying to reach node $u$. Notice that if this happens, then we can define the set $X_{i+1}=\left(X_{i} \backslash\{u\}\right) \cup\{v\}$. 


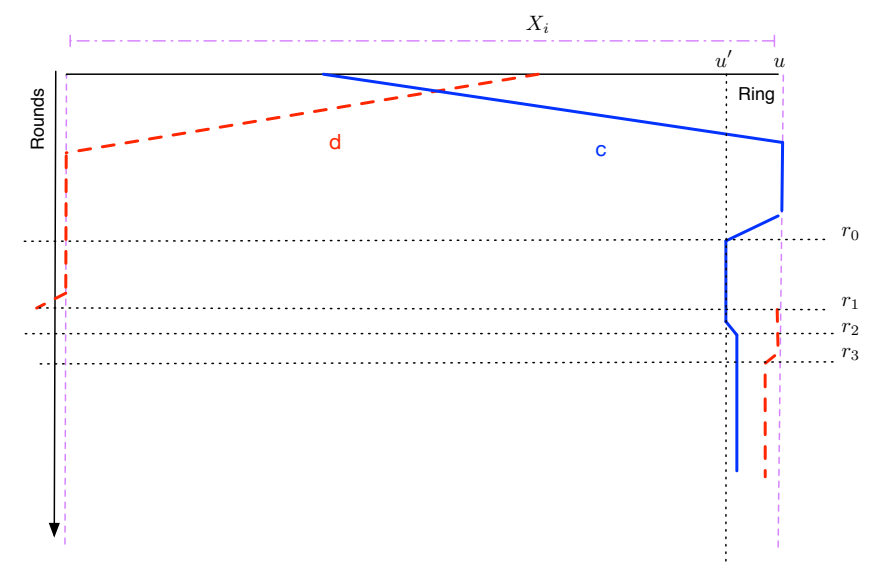

Figure 16: Run where agents end up waiting on the same edge.

If $d$ is blocked traversing an edge to go outside $X_{i+1}$, it cannot wait forever, otherwise the adversary can create a schedule and a configuration in which both agents are forever blocked on the endpoints of edge $\left(u, u^{\prime}\right)$, without the possibility of termination.

An example is shown in Figure 16, in this run we have that agent $c$ is blocked until round $r_{0}$, when it decides to change direction. When $c$ reaches $u^{\prime}$ at round $r_{1}$, it is forced to sleep and agent $d$ is allowed to move reaching $u$. When this happens, agents $c$ is activated again and it tries to traverse edge $\left(u, u^{\prime}\right)$ at round $r_{2}$. At round $r_{3}$, also agent $d$ tries to traverse edge $\left(u, u^{\prime}\right)$, and both agents keep doing that forever without terminating. Notice, that the agents only know an upper bound $N$ on the ring size.

Therefore, agent $d$ eventually leaves node $v$ to go to the neighbouring node in $X_{i+1}$, thus the adversary can let $c$ visit node $u$, then it forces $c$ to sleep and it keeps $d$ blocked until $d$ leaves $v$. At this point we are again in the situation where both agents are confined in the set of nodes $X_{i}=\left(X_{i+1} \backslash\{v\}\right) \cup\{u\}$. If the agents keep behaving in this way, the adversary makes them oscillating forever between the sets $X_{i}$ and $X_{i+1}$.

Therefore, $c$ has to perform $\left|X_{i}\right|-1$ steps and eventually has to reach $d$.

As soon as $c$ performs $\left|X_{i}\right|-1$ additional moves, the adversary starts the next phase $i+1$, selecting $X_{i+1}$ to be the area currently delimited by the blocked edge on one side and the passive agent on the other (a "shift" of the former $X_{i}$ area in the direction of $d$ 's move); that is, $X_{i+1}=\left(X_{i} \backslash\{u\}\right) \cup\{v\}$; thus, $\left|X_{i}\right|=\left|X_{i+1}\right|=x$. Observe that the added node $v$ could have been already visited in a previous round; that is $N_{i} \leq N_{i+1} \leq N_{i}+1$.

The adversary repeats the same process until phase $j$ where $N_{j}=N$. Note that termination cannot occur before the end of this process. In fact, should one agent terminate at any time during the $i$-th phase, $i<j$, then every attempt of the other agent to move would be blocked by the adversary, preventing further exploration, and the algorithm would fail on rings of size $n=N>N_{i}$.

Since the explored part grows of at most one node in each phase $i<j$, the total number of phases is at least $N-x$. Since in each phase at least $x$ moves are performed by the agents, the total number of moves performed by the agents is at least $x(N-x)$. By choosing $x=\lceil n / 2\rceil$ the adversary ensures the claimed bound.

\section{B. Chirality and Landmark}

Consider now the case when, instead of knowledge of an upper bound on the ring size, there is a landmark in the ring.

The general strategy for two agents with chirality (but without bound on the ring size) is essentially the same as the one for PTBoundWithChirality, where however an agent cannot use $N$ for termination, but terminates when it performs a complete loop around the landmark (and thus knows $n$ ), or 


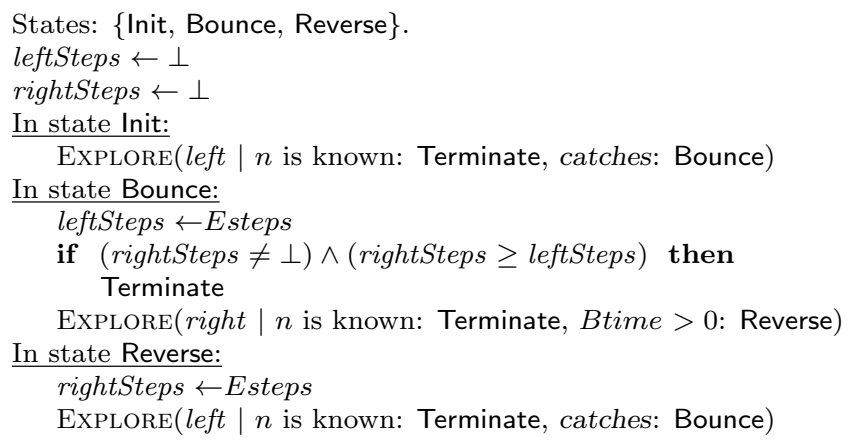

Figure 17: Algorithm PTLANDMARKWithChirality

when it detects that the agents have crossed (like in the previous algorithm). The resulting algorithm PTLANDMARKWITHChIRALiTY is however more efficient in terms of number of link traversals by the agents.

Theorem 14. Two agents executing Algorithm

PTLANDMARKWITHCHIRALITY in the PT model with chirality will explore a ring with a landmark using at most $O\left(n^{2}\right)$ edge traversals. Furthermore, one agent explicitly terminates, while the other either terminates or it waits perpetually on a port.

Proof. The correctness proof is analogous to the one of Theorem 12. Regarding complexity, the key observation is that, in a Bounce-Reverse phase an agent cannot do more than $\mathcal{O}(n)$ steps, otherwise it will loop around the landmark and terminate. Each time rightSteps < leftSteps is verified by an agent, the agent performs an additional step to the left; if this condition is verified $2 n-1$ times, that agent has done a loop around the landmark. Hence the $\mathcal{O}\left(n^{2}\right)$ bound trivially follows.

Adapting the proof of Theorem 13 , it is possible to show that the algorithm is asymptotically optimal:

Theorem 15. In the PT model, any exploration algorithm for a ring of size $n$, with $n \geq 5$, with a landmark by two agents with chirality requires $\Omega\left(n^{2}\right)$ edge traversals in the worst case.

Proof. Let $\mathcal{A}$ be a solution algorithm for exploration of a ring with a landmark by two agents with chirality. Initially the adversary locates both agents at the landmark node and lets them execute the algorithm until $N_{1}=5$ nodes have been explored. The adversary operates in logical phases; let $X_{i}$ denote the area of the ring explored by the agents at the beginning of phase $i$, and let $N_{i}=\left|X_{i}\right|$. In phase $i$ with $N_{i}<n$, the adversary considers one agent at a time. If that agent, say $a$, tries to leave $X_{i}$, the adversary will block that edge and keeps it blocked until either $a$ changes direction (i.e., stays within $X_{i}$ ) or also the other agent tries to leave $X_{i}$ (from opposite directions). Eventually both agents must want to leave $X_{i}$ from opposite directions: otherwise the adversary can continue to prevent the agents from leaving $X_{i}$ and since $N_{i}<n$, the algorithm $\mathcal{A}$ would fail.

When the adversary determines that also the second agent, say $b$, would want to leave $X_{i}$ should it become active (recall the first agent is currently waiting on a blocked edge), the adversary considers what each agent would do, according to $\mathcal{A}$, if the edge the agent wants to cross is blocked indefinitely.

Clearly, they cannot both wait indefinitely, otherwise in the ring where $n=N_{i}$, the adversary, activating both agents at all times, would make the algorithm fail by having the two agents wait forever at the two endpoints of the same blocked edge, without either of them terminating (although they have explored the ring). This means that at least one of the agents, after a finite number of activations, must change direction and move towards a neighbouring node in $X_{i}$. Let $c \in\{a, b\}$ be such an agent, and $d$ be the other. 
Upon this determination, if $d=b$, the adversary activates $d$ (letting it move), and then makes $d$ passive; otherwise, if $d=a$, it makes $a$ passive immediately and let it move passively on the next node.

In any case, it also blocks the edge from which $c$ was trying to leave $X_{i}$ and keeps it blocked until $c$ performs at least $\left|X_{i}\right|$ moves, possibly reaching $d$. Note that, as in the case of Algorithm PTBOundWithCHIRALITY, this will always happen as shown by the claim below.

Claim 2. If $c$ never reaches $d$ in this phase and it does not wait forever in the port of node $u^{\prime}$ trying to reach node $u$, it will perform an unbounded number of moves.

Proof. By contradiction, let $c$ satisfy the conditions of the claim, but perform only a bounded number of moves, without reaching $d$. Thus, within finite time it will stop in a node indefinitely. When this happens, the adversary activates $d$ blocking any edge through which $c$ wants to move. Hence no new node will be explored; since $N_{i}<n$ the adversary would make the algorithm $\mathcal{A}$ fail.

Further note that, during this time, agent $d$ moves (if $d=b$ ) or is passively moved (if $d=a$ ) to a neighbour $v$ outside $X_{i}$.

In Claim 2 we assumed that $c$ does not wait forever in the port of node $u^{\prime}$ trying to reach node $u$. Notice that if this happen, then we can define the set $X_{i+1}=\left(X_{i} \backslash\{u\}\right) \cup\{v\}$, if $d$ is blocked traversing an edge to go outside $X_{i+1}$ it cannot wait forever or the adversary can create a schedule and a configuration in which both agents are forever blocked on the endpoints of edge $\left(u, u^{\prime}\right)$, without the possibility of termination. Therefore, agent $d$ eventually leaves node $v$ to go to the neighbour node in $X_{i+1}$, thus the adversary can let $c$ visit node $u$, it then forces $c$ to sleep and it keeps $d$ blocked until $d$ leaves $v$. At this point we are again in the situation where both agents are confined in the set of nodes $X_{i}=\left(X_{i+1} \backslash\{v\}\right) \cup\{u\}$. If the agents keep behaving in this way, the adversary makes them oscillating forever between the sets $X_{i}$ and $X_{i+1}$.

Therefore, $c$ has to perform $\left|X_{i}\right|-1$ steps and eventually has to reach $d$.

As soon as $c$ performs $\left|X_{i}\right|$ moves, the adversary starts the next phase $i+1$. Since $v$ is a newly explored node, $X_{i+1}$ is the former $X_{i}$ area augmented by node $v$; thus, $N_{i+1}=N_{i}+1$.

The adversary repeats the same process until phase $j$ when $N_{i}=n-1$. Note that termination cannot occur before the end of this process. In fact, should one agent terminate at any time during the $i$-th phase, $i<j$, then every attempt of the other agent to move would be blocked by the adversary, preventing further exploration, and the algorithm would fail because the ring has not been fully explored yet.

Since the explored part grows of one node in each phase, the total number of edge traversals is $\sum_{i=3}^{n} i>\frac{n^{2}}{2}$ and the theorem follows.

\subsubsection{PT: Without Chirality}

Without chirality, we have shown that two agents do not suffice (Theorem 10). We thus consider the presence of three agents in absence of chirality. Also in this case, we need to assume some other knowledge because the presence of three agents without additional information is not sufficient (Theorem 2) even for partial termination.

As in the previous Section, we first assume the agents have knowledge of an upper bound $N$ on the ring size; the case when there exists a landmark node is considered later.

\section{A. Upper Bound}

The algorithm, PTBoundNoChirality, for this case is described in Figure 18. Upon activation, at least two of the three agents will necessarily agree on the orientation. An agent bounces only when catching another agent, performing a "zig-zag tour". There are several ways in which an agent terminates.

More precisely, an agent changes direction if and only if it reaches another agent that is waiting on a missing edge in the same direction.

Each agent memorizes the distance $d$ that it has traveled between the first time it changed state from Bounce to Reverse. Each time the agent changes state from Bounce to Reverse (or viceversa) it checks that 
States: $\{$ Init, Bounce, Reverse,MeetingR, MeetingB\}.

$d \leftarrow 0$

In state Init:

EXPLORE (left | Tnodes $\geq N$ : Terminate, catches: Bounce)

In state Bounce:

CHECKD(Esteps)

EXPLORE(right $\mid$ Tnodes $\geq N$ : Terminate, meeting: MeetingB, catches: Reverse)

In state Reverse:

if $d=0$ then

else

$d \leftarrow$ Esteps

$\triangleright$ First time I change state from Bounce to Reverse

CHeckD(Esteps)

Explore(left $\mid$ Tnodes $\geq N$ : Terminate, meeting: MeetingR, catches: Bounce)

In state MeetingR:

if Esteps $\leq d$ then

Terminate

ExplorenoResetEsteps(left $\mid$ Tnodes $\geq N$ : Terminate, catches: Bounce)

ExPlORE(b)ut it does not reset Esteps

In state MeetingB:

if Esteps $\leq d$ then

Terminate

EXPloReNoResetEsteps(right $\mid$ Tnodes $\geq N$ : Terminate, catches: Reverse)

EXPLORE(b)ut it does not reset Esteps

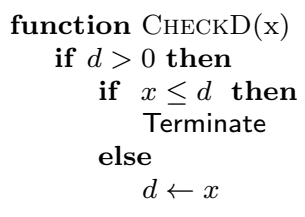

$\triangleright$ This procedure is the same of

$\triangleright$ This procedure is the equal of

Figure 18: Algorithm PTBoundNoChIRALITY

the number of steps done is strictly greater than $d$, otherwise it terminates. If the distance is greater the agent memorizes this new distance. If the agent meets an agent on a node (condition MeetingB and MeetingR of the algorithm) and distance $d$ has been set, it also checks that the distance from the last direction change is greater than $d$ and updates $d$, otherwise it terminates. Finally, there is a trivial terminating condition of doing $N$ steps in the same direction.

Lemma 4. In Algorithm PTBoundNoChirality, if an agent terminates then the ring has been explored.

Proof. The only non trivial part is the termination for Condition [if Esteps $\leq d$ ]. Let $b$ be the first agent that terminates by satisfying condition Esteps $\leq d$ (if there are several such agents, take any of them), at some round $r_{2}$. We will show that assuming that there are unexplored nodes in the ring at time $r_{2}$ leads to contradiction.

First note that, if there are unexplored nodes at time $r_{2}$, no agent could have terminated before time $r_{2}$ by satisfying condition Tnodes $\geq N$; i.e., $b$ is indeed the first agent to terminate.

Let us define rounds $r_{0}$ and $r_{1}$ as the last two rounds in which $b$ changed direction, with $r_{0}<r_{1}$ (see Figure 19). Observe that $r_{0}$ and $r_{1}$ are well defined: At time $r_{2}, b$ has a positive value of $d$ (otherwise Esteps $\leq d$ won't be satisfied). By construction of the algorithm, the first change of direction does not set $d$, only the subsequent ones do; i.e., $b$ must have had at least two changes of direction before satisfying the termination condition.

Let us define the leftmost and the rightmost agents as follows:

- If at time $r_{0}$ agent $b$ changes direction from left to right, then agent $b$ becomes the rightmost agent, while agent $a$ (that $b$ catched and reversed direction on) becomes the leftmost agent. Otherwise, $b$ becomes the leftmost agent and $a$ becomes the rightmost agent. 


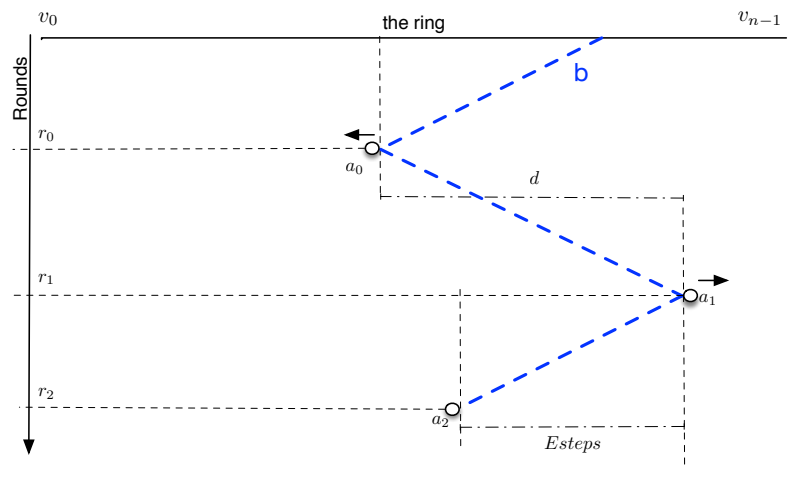

Figure 19: PTNoChirality Termination

- The leftmost agent remains so unless it is overtaken by another agent $a^{\prime}$ moving left or it bounces on an agent $a^{\prime \prime}$, in which case the other agent becomes the leftmost agent.

- Similarly, the rightmost agent remains so unless it is overtaken by another agent $b^{\prime}$ moving right or it bounces on an agent $b^{\prime \prime}$, in which case the other agent becomes the rightmost agent.

Let us define as visited (for any round $r, r_{0} \leq r \leq r_{2}$ ) the set of nodes which have been visited by the leftmost or the rightmost agent by round $r$ (included). A node that is not visited at round $r$ is called unvisited at round $r$. Hence, if there are no unvisited nodes, the whole ring has been explored. It is easy to show by induction on the round number, that the set of visited nodes forms a compact set of nodes between the leftmost and the rightmost agent (it starts as a single node at time $r_{0}$, and grows only by leftward move of the leftmost agent and by a rightward move of the rightmost agent; note that the fact that the actual leftmost/rightmost agents might change over time does not affect this). Hence, the leftmost and the rightmost visited node are well defined.

From the definition of the leftmost/rightmost agents (in particular, that the leftmost/rightmost agent never disappears, it can only be replaced by another one) it follows that as long as there are unvisited nodes (i.e. by assumption up to and including round $r_{2}$ ), the leftmost visited node is occupied by the leftmost agent and the rightmost visited node is occupied by the rightmost agent. As there are three agents altogether, this means that the internal visited nodes (different from the leftmost and the rightmost one) contain together at most one agent. As change of direction and/or test Esteps $\leq d$ are performed only when an agent catches another agent, as long as there are unvisited nodes, no agent can change direction or terminate due to Esteps $\leq d$ in an internal visited node.

Hence, when $b$ changed direction in round $r_{1}$, it was in the rightmost or leftmost visited node (depending on its direction in round $r_{0}$ ). As $b$ did not terminate in round $r_{1}, b$ set its $d$ in the last line of function CHECKD to be the distance it traveled between $r_{0}$ and $r_{1}$. As $b$ changed direction in $r_{1}$, after traveling less than $d$ step it is still in an internal visited node and cannot terminate. Hence, $b$ must have terminated in round $r_{2}$ by satisfying Esteps $=d$. However, this means that the leftmost agent did not advance in any round between $r_{0}$ and $r_{2}$. In particular, it did not advance in round $r_{1}$, which is only possible (due to passive transport) if both leftmost and rightmost agents were blocked on a single edge. That is, all the nodes have already been visited in round $r_{1}$, a contradiction.

Theorem 16. Three agents performing Algorithm

PTBOUNDNOCHIRALITY in the PT model with a known upper bound $N$ on the ring size and no chirality, explore the ring with $O\left(N^{2}\right)$ edge traversals. One agent explicitly terminates, the others either terminate or wait perpetually on a port.

Proof. The correctness of termination derives from Lemma 4 It remains to prove that eventually at least one agent terminates. Having three agents, at least two will agree on the same direction. We will consider this 
direction as global left. It is easy to see that if an edge is perpetually removed, then eventually the agents terminate: two agents will be positioned at the end point of the missing edge and the third agent terminates detecting Esteps $=d$. If an agent is not forced to change direction and the edges are not perpetually removed, then it will terminate since Esteps $>N$. Therefore, the adversary has to force the agents to bounce on each other. But let us notice that, as soon as an agent changes state from Bounce to Reverse, it sets a distance $d$; if this distance does not increase at each state change, the agent terminates. This implies that eventually we will have $d>N$ and termination for Esteps $>N$.

Let us now analyze the complexity of the algorithm. If an agent does not set $d$, then it performs at most $O(N)$ steps. If an agent sets $d$, its value is at most $O(N)$; there are at most $O(N)$ increases of $d$, therefore an agent will do at most $O\left(N^{2}\right)$ movements. Since the number of agents is constant, the total sum of movements over all agents is at most $O\left(N^{2}\right)$.

\section{B. Landmark}

The general strategy for three agents without chirality and without an upper bound on the ring size, Algorithm PTLANdmarkNoChirality, is essentially the same as the one for Algorithm PTBoundNoChirality, where however an agent cannot use $N$ for termination, but terminates when it performs a complete loop around the landmark (and thus knows $n$ ), or when it detects that the agents have crossed (like in the previous algorithm). Essentially, Algorithm

PTLANDMARKNoChIRALITY is obtained by modifying Algorithm PTBoundNoChirality (shown in Figure 18) as follows: the predicate "Tnodes $\geq N$ " is substituted with " $n$ is known", that is the agent has done a loop around the landmark.

Theorem 17. Three agents performing Algorithm

PTLANDMARKNOCHIRALITY in the PT model with no chirality in presence of a landmark, explore the ring with $O\left(n^{2}\right)$ edge traversals. One agent explicitly terminates, the others either terminate or wait perpetually on a port.

The proof follows the same lines of the one of Theorem 16. where termination does not happens when Esteps $\geq N$ but the first time an agent does a loop around the landmark. It is easy to see that this has to happen since $d$ increases.

\subsection{Exploration in the ET Model}

\subsubsection{Basic Results}

Let us first introduce a simple result on a unconscious exploration:

Theorem 18. In the ET model with chirality, two robots can perform an unconscious exploration of the ring.

Proof. A trivial algorithm in which an agent changes direction only when it catches someone solves the exploration in ET.

Given the previous results, a natural question is whether there is an algorithm with partial termination, as we have shown for the PT model. Unfortunately the following theorem shows that, without exact knowledge of the network size, it is impossible to design such an algorithm.

Theorem 19. Let us consider the ET model where only an upper bound on the ring size is known. Given any number of agents, there does not exist any exploration algorithm with partial termination. This holds true even if the ring has a landmark node, the agents have distinct IDs, there is common chirality.

Proof. The proof is by contradiction. Let us assume that there is an exploration algorithm $\mathcal{A}$ that always achieves partial termination under the assumptions of the theorem. Let us consider two different rings $R_{1}=\left(V_{1}, E_{1}\right)$ and $R_{2}=\left(V_{2}, E_{2}\right)$, with $V_{1}:\left\{v_{0}, \ldots, v_{n-1}\right\}$ and $V_{2}:\left\{v_{0}, \ldots, v_{n^{\prime}-1}\right\}$ and where $n<n^{\prime}$. Let $e_{i}$ 
denote the edge $\left(v_{i}, v_{i+1}\right)$, where indices are taken modulo the size of the ring. Finally, let $N$ be the upper bound on the both ring sizes such that $n^{\prime}<N$.

On ring $R_{1}$, starting from round 0 , the adversary perpetually removes edge $e_{0}$, and it schedules the activation of the agents as follows. In rounds where the agents would not traverse $e_{0}$ or they would traverse $e_{0}$ from one endpoint only, the adversary lets all the agents be active. In the busy rounds where there are agents trying to traverse $e_{0}$ from both endpoints, say nodes $v_{0}$ and $v_{n-1}$, the adversary activates agents on $v_{0}$ and on $v_{n-1}$ in alternating fashion, i.e. they are never active in the same round. Notice that $e_{0}$ is perpetually removed, so no one will traverse it. Since $\mathcal{A}$ is assumed to be correct, it will eventually explore $R_{1}$. Therefore, there exists a round $r$ where $\mathcal{A}$ decides that $R_{1}$ has been explored, and at least one agent terminates.

We now consider the following schedule of activations and edges removal for ring $R_{2}$. On ring $R_{2}$ the adversary places all agents in the same positions of ring $R_{1}$, i.e. they are only in nodes belonging to $\left\{v_{0}, v_{1} \ldots, v_{n-1}\right\}$. In rounds when some agent wants to traverse one of edges $e_{0}$ and $e_{n}$ and no agent wants to traverse the other, the adversary will block that edge. In the busy rounds when there are agents trying to traverse both $e_{0}$ and $e_{n}$, it will alternate between making agents waiting on $e_{0}$ passive and blocking edge $e_{n}$, and making agents waiting on $e_{n}$ passive and blocking $e_{0}$. Notice that, in the ET model, such a schedule can be kept for a finite but unbounded amount of rounds, hence this can be kept until round $r$.

Observe that, until round $r$, this execution of $\mathcal{A}$ on $R_{2}$ is not distinguishable, from the point of view of agents, from the execution of $\mathcal{A}$ on $R_{1}$ previously explained. Therefore, the agent that terminates at round $r$ in the execution of $\mathcal{A}$ on $R_{1}$ also terminates in round $r$ in the execution of $\mathcal{A}$ on $R_{2}$. However, when this happens ring $R_{2}$ has not been explored.

Note that the above arguments apply also if the agents have distinct IDs. If there is a landmark, it can be placed anywhere in $R_{1}$, and in the corresponding node in $R_{2} ; R_{1}$ and $R_{2}$ will still look the same to the agents.

Notice that Theorem 19 would hold even if the agents were equipped with wireless communication.

\subsubsection{Exploration Algorithm for ET}

Algorithm ETBoundNoChiRALiTy is a direct adaptation of Algorithm PTBoundNoChiRALiTy, the only differences are that $N$ is set to $n-1$ (since from Theorem 19 we know the size needs to be known precisely), and the inequality check in CheckD becomes strict: (if Esteps $<d$ ). As in the PT model, three agents are employed, with no chirality assumption.

Theorem 20. Three anonymous agents performing Algorithm ETBOundNoCHIRALITY in the ET model with known ring size and no chirality explore the ring, with one agent explicitly terminating and the other agents either terminating or waiting perpetually on a port.

Proof. Let us first observe that if an agent terminates, then it terminates correctly. The proof follows the same steps as the one for Lemma 4. The only difference is that in ET the CheckD requires (if Esteps $<d$ ), thus the part of the proof that uses the PT assumption to handle the case Esteps $=d$ is not needed anymore.

What remains to be shown is that eventually at least one agent terminates. We show this by contradiction. Let us notice that if an edge is perpetually removed, an agent eventually terminates: two agents will be positioned at the two ports of the missing edge and the other one will do exactly Esteps $=n-1$ steps going from one endpoint node to the others. If an edge is not perpetually removed then, by construction (the agents bounce only on other agents, not on timeout on blocked edges) and by the ET condition, an agent that is waiting on the port of that edge will eventually traverse it. Since the agents terminate if they traverse $n-1$ steps in the same direction, the only possibility left to consider is for the adversary to force the agents to perpetually catch each other, changing directions without increasing the $d$ value. In such a case there is a round $r^{*}$ after which each agent $x$ reaches a certain stable value $d_{x}$ and it always changes position at the same points $l_{x}$ and $r_{x}$; furthermore, there exists a round $r \geq r^{*}$ in which two agents go left and one agent goes right. In the remainder we assume the execution is in round at least $r$. 

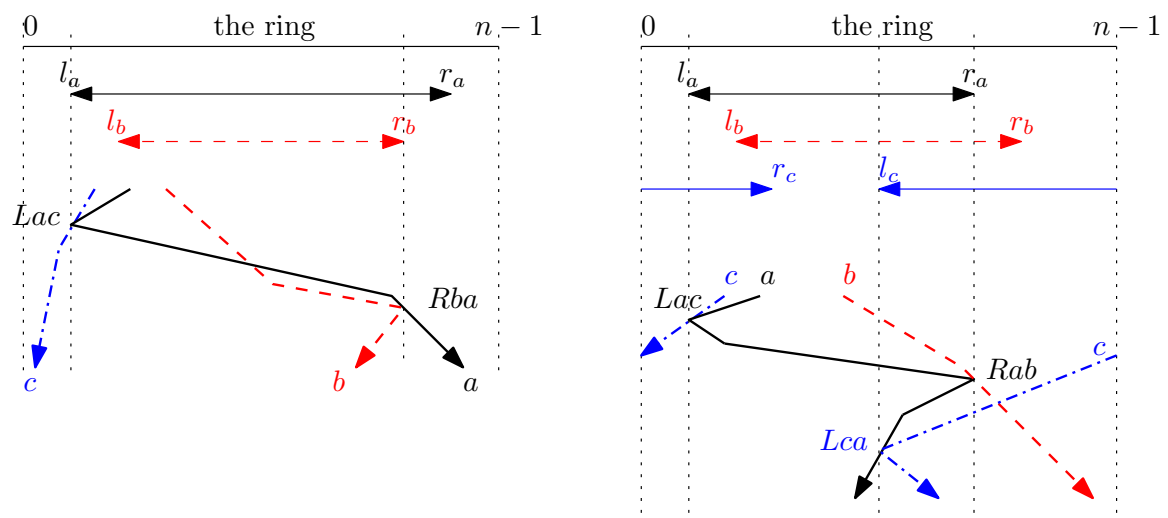

Figure 20: Left: The agent ranges and events for the case Lac: Rba. Right: the case Lac: Rab.

Let $a, b, c$ be the agents and let $L x y$ indicate the event: agent $x$ catches agent $y$ at $l_{x}$ (and changes direction from left to right); the event $R x y$ is analogous. Let $D x y: D^{\prime} x^{\prime} y^{\prime}$ denote the statement that the event $D^{\prime} x^{\prime} y^{\prime}$ is the catching event immediately following $D x y$.

Note that the $D x y$ event can only be followed by an $\bar{D} x z$ or $\bar{D} z x$ events (here and in the remainder, we implicitly assume $x \neq y \neq z$ ), where $D \in\{L, R\}$ and $\bar{D}$ is the direction opposite to $D$. This is so because, after $D x y$, agents $x$ and $z$ were moving in the same direction $\bar{D}, y$ remained moving in direction $D$, and only agents that move in the same direction can catch each other. Hence, the possible sequences of direction changes of a perpetual schedule can be represented by a binary tree rooted at the initial Dxy event (w.l.o.g. $L a b$ or $L a c$ ), with leaves corresponding to agent termination. We call this tree Catch Tree, see Figure 22. The assumption that the algorithm does not terminate implies that there is an infinite path in this tree. In the remainder of this proof we will show that there is no such path in the Catch Tree.

First, observe that the agents never meet on a node - that would imply at least one of them performing the termination check somewhere inside its range, immediately terminating. This means that unlike the case of Algorithm PTBoundNoChiRALITY we do not have to worry about the agents overtaking each other. (They can still cross each other without noticing when traveling in opposite direction and crossing the same edge at the same time.)

Consider now a sequence of events $D x y: \bar{D} x z: D x y$. This corresponds to $x$ bouncing off $y$ and $z$ while those remained stationary. From the ET condition and the fact that $y$ and $z$ are waiting on different edges (otherwise $x$ would detect $d=n-1$ and terminate) we know that eventually either $y$ or $z$ makes progress, hence this sequence cannot repeat indefinitely and eventually a different event must occur. This can be represented in the Catch Tree by removing the bottom Dxy (and its whole subtree) and adding an arrow from its parent to the top $D x y$ (implying that the same configuration repeats). For a representation of this see Figure 22

Let $A, B, C$ be the ranges (sets of nodes an $x$ agent visits between $l_{x}$ and $r_{x}$ ) of agents $a, b, c$, respectively, and $\bar{A}, \bar{B}, \bar{C}$ be their complements.

Claim 3. If the algorithm does not terminate, we have $\forall X, Y \in\{A, B, C\}, X \neq Y: \bar{X} \cap \bar{Y}=\emptyset$.

Proof. The proof is by contradiction. Assume w.l.o.g, $A, B$ such that $\bar{A} \cap \bar{B} \neq \emptyset$. From this and symmetry, we may assume $l_{a} \notin B$. This means $L a c$ is the only way for the agent $a$ to change direction while going left. Consider the possible next event (consult Figure 20 left). It cannot be $R b a$ : for that $r_{b}$ must be in $A$, which combined with $l_{a} \notin B$ implies $\bar{A} \subset \bar{B}$. Since from the moment of Lac agent $a$ was moving right, starting outside $B$ and to the left of it, in order to bounce off $b$ it would need to overtake it, which is impossible.

Hence, $L a c$ must be followed by Rab, which in turn might be followed by Lac or Lca. Lac is the cyclic case which, as has been discussed above, cannot repeat indefinitely and can therefore be ignored. The only possible case is $L c a$, which can only happen if $\bar{C} \subset A$. 


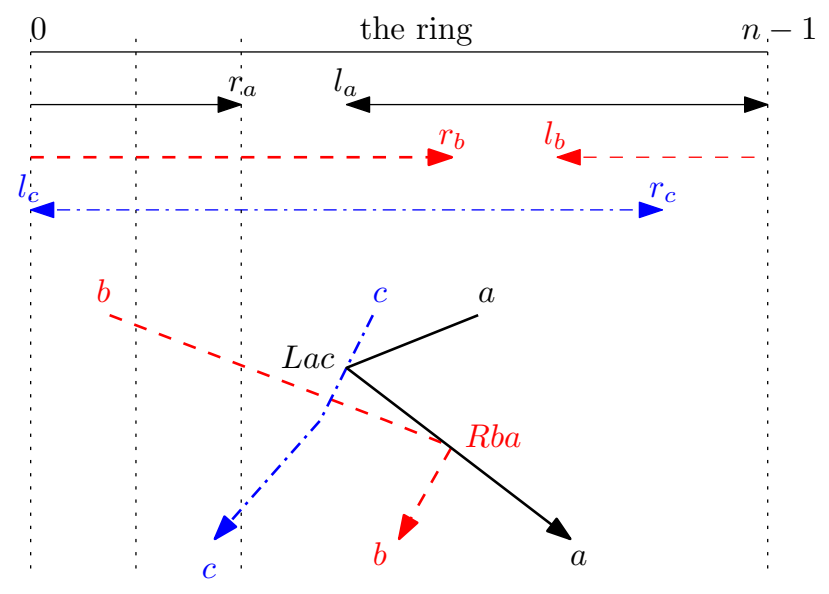

Figure 21: The case of $L a c: R b a$ for disjoint range complements.

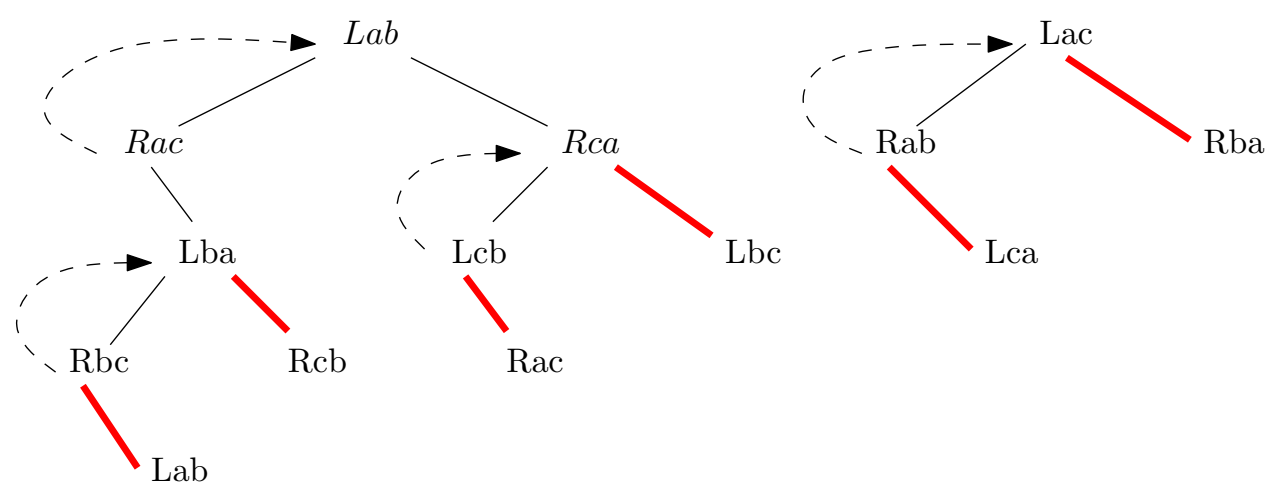

Figure 22: Catch Trees rooted at $L a b$ and $L a c$. The red edges denote forbidden sequences, while the dashed ones depict the loops.

Observe the movement of the agents (see Figure 20 right): From Rab on, $b$ is moving right from $r_{a}$, while from $L c a$ on, $c$ is moving right from $l_{c}$. This means that $R b c$ is impossible, because $c$ would have had to overtake $b$. However, $R c b$ is also impossible as $b$ cannot get into position at $r_{c}$ without changing direction or crossing $\bar{B}$.

From Claim 3 it remains to consider the case of pairwise disjoint range complements, as shown in Figure 21 .

Claim 4 If the algorithm never terminates, and if the agents are named in such a way that the complement of the ranges are in order $\bar{A}, \bar{B}, \bar{C}$ from left to right (see Figure 21), then Lac cannot be immediately followed by $R b a$.

Proof. After event $L a c$, we have that $c$ is moving left from $l_{a}$. After event $R b a$, agent $b$ is moving left from $r_{b}$. The next event is either $L b c$ or $L c b$. However, $L b c$ is impossible, as for $c$ to reach $l_{b}$ without changing direction it would have to cross $\bar{C}$. Event $L c b$ is also impossible, because $b$ would have to overtake $c$ to get into a position where $c$ could bounce on $b$.

The following corollary is immediately obtained from Claim 4 by rotation and symmetry:

Claim 5 If the algorithm never terminates, then the following sequences are forbidden Lac: Rba, Lba: Rcb, $L c b: R a c, R b c: L a b, R c a: L b c, R a b: L c a$. 
At some point in the perpetual schedule we should have either $L a b$ or $L a c$. As can be seen in Figure 22 , any branch of a Catch Tree starting from these configuration leads either to a cycle or to a forbidden sequence of events, leading to a contradiction with the assumption that the algorithm never terminates.

Note that the number of moves performed by the agents before termination is finite but possibly unbounded. Consider the situation when two agents are blocked going on opposite directions on two different edges, while the third agent goes back and forth between them; since we are in the ET model, this configuration cannot be kept forever, but there is no bound on the number of rounds in which it holds.

\section{Conclusion}

In this paper we started the investigation of the distributed exploration problem for 1-interval-connected dynamic graphs by focusing on rings in fully synchronous and semi-synchronous environments. We studied the impact that structural information and knowledge can have on the solvability of the problem. In particular, we considered such factors as: knowledge of the exact size of the ring or of an upper bound, agreement on orientation, anonymity.

These results open the investigation of live exploration of dynamic networks and the algorithmic study of dynamic networks in the semi-synchonous environment.

Among the several open problems, a challenging one is the study of live exploration in a network of arbitrary topology where, right now, almost nothing is known. In particular, there are no non-trivial bounds on the number of agents, exploration time and type of knowledge necessary for solvability. Little is also known in case of dynamic networks with special but relevant underlying topologies, such as meshes, tori, hypercubes, etc.

A parallel line of research could be to provide formal proofs of that can be automatically verified by theorem provers. This seems particularly important for the case of distributed algorithms working on dynamic networks since, besides the inherent difficulties of designing a distributed algorithm, there is the additional non trivial components of considering all possible dynamic graphs.

Finally, it would be interesting to study distributed solutions to other classical problems for mobile agents (such as gathering, in this regard see the recent 22, and pattern formation) in dynamic networks.

\section{Acknowledgments.}

We would like to thank the anonymous reviewers for the comments and suggestions. Especially, for suggesting the improved bounds discussed in Section 3.1 and a correction of Theorem 5 .

This research has been supported in part by NSERC under the Discovery Grant program, by Dr. Flocchini's University Research Chair, and by the VEGA 2/0165/16 grant.

\section{References}

[1] Albers, S., Henzinger, M.: Exploring unknown environments. SIAM Journal on Computing 29(4), $1164-1188(2000)$

[2] Arantes, L., Greve, F., Sens, P., Simon, V.: Eventual leader election in evolving mobile networks. In Proc. of the 17th Int. Conference on Principles of Distributed Systems (OPODIS) pp. 23-37 (2013)

[3] Augustine, J., Pandurangan, G., Robinson, P.: Fast byzantine agreement in dynamic networks. In Proc. of the 32th Symposium on Principles of Distributed Computing (PODC) pp. 74-83 (2013)

[4] Avin, C., Koucky, M., Lotker, Z.: How to explore a fast-changing world. In Proc. of the 35th International Colloquium on Automata, Languages and Programming (ICALP) pp. 121-132 (2008) 
[5] Awerbuch, B., Even, S.: Efficient and reliable broadcast is achievable in an eventually connected network. In Proc. of the 3th Symposium on Principles of Distributed Computing (PODC) pp. 278-281 (1984)

[6] Balamohan, B., Dobrev, S., Flocchini, P., Santoro, N.: Exploring an unknown dangerous graph with a constant number of tokens. Theoretical Computer Science 610, 169-181 (2016)

[7] Baumann, H., Crescenzi, P., Fraigniaud, P.: Parsimonious flooding in dynamic graphs. Distributed Computing 24(1), 31-44 (2011)

[8] Biely, M., Robinson, P., Schmid, U.: Agreement in directed dynamic networks. In Proc. of the 19th International Colloquium on Structural Information and Communication Complexity (SIROCCO) pp. 73-84 (2012)

[9] Brejova, B., Dobrev, S., Kralovic, R., Vinar, T.: Efficient routing in carrier-based mobile networks. Theoretical Computer Science 509, 113-121 (2013)

[10] Bui-Xuan, B., Ferreira, A., Jarry, A.: Computing shortest, fastest, and foremost journeys in dynamic networks. International Journal of Foundations of Computer Science 14(2), 267-285 (2003)

[11] Casteigts, A., Flocchini, P., Mans, B., Santoro, N.: Measuring temporal lags in delay-tolerant networks. IEEE Transactions on Computers 63(2), 397-410 (2014)

[12] Casteigts, A., Flocchini, P., Mans, B., Santoro, N.: Shortest, fastest, and foremost broadcast in dynamic networks. International Journal of Foundations of Computer Science 25(4), 499-522 (2015)

[13] Casteigts, A., Flocchini, P., Quattrociocchi, W., Santoro, N.: Time-varying graphs and dynamic networks. Int. Journal of Parallel, Emergent and Distributed Systems 27(5), 387-408 (2012)

[14] Chalopin, J.: Election and rendezvous with incomparable labels. Theoretical Computer Science 399(1), $54-70(2008)$

[15] Clementi, A., Monti, A., Pasquale, F., Silvestri, R.: Information spreading in stationary markovian evolving graphs. IEEE Transactions on Parallel and Distributed Systems 22(9), 1425-1432 (2011)

[16] Cooper, C., Klasing, R., Radzi, T.: Searching for black-hole faults in a network using multiple agents. In Proc. of the 10th International Conference on Principles of Distributed Systems (OPODIS) pp. 320-332 (2006)

[17] Das, S., Flocchini, P., Kutten, S., Nayak, A., Santoro, N.: Map construction of unknown graphs by multiple agents. Theoretical Computer Science 385(1-3), 34-48 (2007)

[18] Deng, X., Papadimitriou, C.H.: Exploring an unknown graph. Journal of Graph Theory 32(3), 265-297 (1999)

[19] Di Luna, G., Baldoni, R.: Brief announcement: Investigating the cost of anonymity on dynamic networks. In Proc of the the 34th Symposium on Principles of Distributed Computing (PODC) pp. 339-341 (2015)

[20] Di Luna, G., Baldoni, R., Bonomi, S., Chatzigiannakis, I.: Conscious and unconscious counting on anonymous dynamic networks. In Proc. of the 15th International Conference on Distributed Computing and Networking (ICDCN) pp. 257-271 (2014)

[21] Di Luna, G., Dobrev, S., Flocchini, P., Santoro, N.: Live exploration of dynamic rings. In Proc. of the 36th IEEE International Conference on Distributed Computing Systems (ICDCS) pp. 570-579 (2016)

[22] Di Luna, G., Flocchini, P., Pagli, L., Prencipe, G., Santoro, N., Viglietta, G.: Gathering in dynamic rings. In Proc. of the 24th International Colloquium on Structural Information and Communication Complexity (SIROCCO) pp. 339-355 (2017) 
[23] Dieudonn, Y., Pelc, A.: Deterministic network exploration by anonymous silent agents with local traffic reports. ACM Transactions on Algorithms 11(2), Article No. 10 (2014)

[24] Dobrev, S., Flocchini, P., Kralovic, R., Santoro, N.: Exploring a dangerous unknown graph using tokens. Theoretical Computer Science 472, 28-45 (2013)

[25] Dobrev, S., Flocchini, P., Prencipe, G., Santoro, N.: Searching for a black hole in arbitrary networks: Optimal mobile agents protocolss. Distributed Computing 19(1), 1-19 (2006)

[26] Erlebach, T., Hoffmann, M., Kammer, F.: On temporal graph exploration. In Proc. of 42th International Colloquium on Automata, Languages, and Programming (ICALP) pp. 444-455 (2015)

[27] Ferreira, A.: Building a reference combinatorial model for MANETs. IEEE Network 18(5), 24-29 (2004)

[28] Flocchini, P., Kellett, M., Mason, P., Santoro, N.: Map construction and exploration by mobile agents scattered in a dangerous network. In Proc. of the 24 th IEEE International Parallel and Distributed Processing Symposium (IPDPS) pp. 1-10 (2009)

[29] Flocchini, P., Kellett, M., Mason, P., Santoro, N.: Searching for black holes in subways. Theory of Computing Systems 50(1), 158-184 (2012)

[30] Flocchini, P., Mans, B., Santoro, N.: On the exploration of time-varying networks. Theoretical Computer Science 469, 53-68 (2013)

[31] Flocchini, P., Prencipe, G., Santoro, N.: Distributed Computing by Oblivious Mobile Robots. Morgan \& Claypool (2012)

[32] Fraigniaud, P., Ilcinkas, D., Peer, G., Pelc, A., Peleg, D.: Graph exploration by a finite automaton. Theoretical Computer Science 345(2-3), 331-344 (2005)

[33] Godard, E., Mazauric, D.: Computing the dynamic diameter of non-deterministic dynamic networks is hard. In Proc. of the 10th Symposium on Algorithms and Experiments for Sensor Systems, Wireless Networks and Distributed Robotics (ALGSENSORS) pp. 88-102 (2014)

[34] Harary, F., Gupta, G.: Dynamic graph models. Mathematical and Computer Modelling 25(7), 79-88 (1997)

[35] Ilcinkas, D., Klasing, R., Wade, A.: Exploration of constantly connected dynamic graphs based on cactuses. In Proc. 21st International Colloquium Structural Information and Communication Complexity (SIROCCO) pp. 250-262 (2014)

[36] Ilcinkas, D., Wade, A.: On the power of waiting when exploring public transportation systems. In Proc. 15th International Conference on Principles of Distributed Systems (OPODIS) pp. 451-464 (2011)

[37] Ilcinkas, D., Wade, A.: Exploration of the t-interval-connected dynamic graphs: the case of the ring. In Proc. 20th International Colloquium on Structural Information and Communication Complexity (SIROCCO) pp. 13-23 (2013)

[38] Kuhn, F., Lynch, N., Oshman, R.: Distributed computation in dynamic networks. In Proc. of the 42th Symposium on Theory of Computing (STOC) pp. 513-522 (2010)

[39] Kuhn, F., Moses, Y., Oshman, R.: Coordinated consensus in dynamic networks. In Proceedings 30th Symposium on Principles of Distributed Computing (PODC) pp. 1-10 (2011)

[40] Liu, C., Wu, J.: Scalable routing in cyclic mobile networks. IEEE Transactions on Parallel and Distributed Systems 20(9), 1325-1338 (2009) 
[41] Michail, O., Spirakis, P.G.: Traveling salesman problems in temporal graphs. Theoretical Computer Science 634, 1-23 (2016)

[42] O'Dell, R., Wattenhofer, R.: Information dissemination in highly dynamic graphs. In Proc. of the Joint Workshop on Foundations of Mobile Computing (DIALM-POMC) pp. 104-110 (2005)

[43] Panaite, P., Pelc, A.: Exploring unknown undirected graphs. Journal of Algorithms 33, 281-295 (1999)

[44] Shannon, C.: Presentation of a maze-solving machine. In Proc. of the 8th Conf. of the Josiah Macy Jr. Foundation (Cybernetics) pp. 173-180 (1951) 